Supporting Information for

\title{
Stereoselective Synthesis of the C1-C16 Fragment of Purported Structure of Formosalide B
}

Srinivas Gajula, ${ }^{\dagger}$ Aedula Vishnu V. Reddy, ${ }^{\dagger}+$ D. Prabhakar Reddy, ${ }^{\dagger}$ Jhillu S. Yadav,${ }^{\dagger}$ Debendra K. Mohapatra*,†,

${ }^{\dagger}$ Department of Organic Synthesis and Process Chemistry, CSIR-Indian Institute of Chemical Technology, Hyderabad-500007, India; $\$$ Academy of Scientific and Innovative Research (AcSIR), New Delhi 110025, India; mohapatra@iict.res.in 


\section{Table of Contents}

\begin{tabular}{|c|c|c|}
\hline S. No & Description & Page No. \\
\hline 01 & General information & S04 \\
\hline 02 & $\begin{array}{l}\text { Experimental procedures and data for the } \\
\text { synthesis of } \mathrm{C} 1-\mathrm{C} 16 \text { fragment of Formosalide } \mathrm{B}\end{array}$ & S04-S18 \\
\hline 03 & ${ }^{1} \mathrm{H}$ and ${ }^{13} \mathrm{C}$ NMR Copy of 7 & S19-S20 \\
\hline 04 & ${ }^{1} \mathrm{H}$ and ${ }^{13} \mathrm{C}$ NMR Copy of 9 & $\mathrm{~S} 21-\mathrm{S} 22$ \\
\hline 05 & ${ }^{1} \mathrm{H}$ and ${ }^{13} \mathrm{C}$ NMR Copy of $\mathbf{1 0}$ & S23-S24 \\
\hline 06 & ${ }^{1} \mathrm{H}$ and ${ }^{13} \mathrm{C}$ NMR Copy of $\mathbf{1 1}$ & $\mathrm{S} 25-\mathrm{S} 26$ \\
\hline 07 & ${ }^{1} \mathrm{H}$ and ${ }^{13} \mathrm{C}$ NMR Copy of $\mathbf{1 2}$ & S27-S28 \\
\hline 08 & ${ }^{1} \mathrm{H}$ and ${ }^{13} \mathrm{C}$ NMR Copy of $\mathbf{1 3}$ & S29-S30 \\
\hline 09 & ${ }^{1} \mathrm{H}$ and ${ }^{13} \mathrm{C}$ NMR Copy of $\mathbf{1 5}$ & S31-S32 \\
\hline 10 & ${ }^{1} \mathrm{H}$ and ${ }^{13} \mathrm{C}$ NMR Copy of 5 & S33-S34 \\
\hline 11 & ${ }^{1} \mathrm{H}$ and ${ }^{13} \mathrm{C}$ NMR Copy of $\mathbf{1 6}$ & $\mathrm{S} 35-\mathrm{S} 36$ \\
\hline 12 & ${ }^{1} \mathrm{H}$ and ${ }^{13} \mathrm{C}$ NMR Copy of $\mathbf{1 7}$ & S37-S38 \\
\hline 13 & ${ }^{1} \mathrm{H}$ and ${ }^{13} \mathrm{C}$ NMR Copy of $\mathbf{1 8}$ & S39-S40 \\
\hline 14 & ${ }^{1} \mathrm{H}$ and ${ }^{13} \mathrm{C}$ NMR Copy of 19 & S41-S42 \\
\hline 15 & ${ }^{1} \mathrm{H}$ and ${ }^{13} \mathrm{C}$ NMR Copy of 6 & S43-S44 \\
\hline 16 & ${ }^{1} \mathrm{H}$ and ${ }^{13} \mathrm{C}$ NMR Copy of 4 & S45-S46 \\
\hline 17 & ${ }^{1} \mathrm{H}$ and ${ }^{13} \mathrm{C}$ NMR Copy of $\mathbf{2 0}$ & S47-S48 \\
\hline 18 & ${ }^{1} \mathrm{H}$ and ${ }^{13} \mathrm{C}$ NMR Copy of $\mathbf{3}$ & S49-S50 \\
\hline
\end{tabular}




\begin{tabular}{|c|c|c|}
\hline 19 & $\begin{array}{r}\text { NOESY Spectrum of compound } \mathbf{3}\left(400 \mathrm{MHz}, \mathrm{CDCl}_{3}\right) \\
(2.5 \text { to } 5.0 \mathrm{ppm})\end{array}$ & $\mathrm{S} 51$ \\
\hline 20 & $\begin{array}{c}\text { NOESY Spectrum of compound } \mathbf{3}\left(400 \mathrm{MHz}, \mathrm{CDCl}_{3}\right) \\
(6.0 \text { to } 8.0 \mathrm{ppm})\end{array}$ & $\mathrm{S} 52$ \\
\hline 21 & $\begin{array}{c}\text { NOESY Spectrum of compound } \mathbf{3}\left(400 \mathrm{MHz}, \mathrm{CDCl}_{3}\right)(- \\
0.2 \text { to } 2.2 \mathrm{ppm})\end{array}$ & $\mathrm{S} 53$ \\
\hline 22 & $\begin{array}{c}\text { NOESY Spectrum of compound } \mathbf{3}\left(400 \mathrm{MHz}, \mathrm{CDCl}_{3}\right) \\
\text { Full Spectrum })\end{array}$ & $\mathrm{S} 54$ \\
\hline
\end{tabular}




\section{Experimental procedures and analytical data:}

1.1 General Information: Experiments which required an inert atmosphere were carried out under argon in flame-dried glassware. THF freshly distilled over sodium/benzophenone and transferred via syringe. Dichloromethane was freshly distilled from $\mathrm{CaH}_{2}$. Tertiary amines were freshly distilled over $\mathrm{KOH}$. Commercially available reagents were used as received. Unless detailed otherwise, "workup" means pouring the reaction mixture into brine, followed by extraction with the solvent indicated in parentheses. If the reaction medium was acidic (basic), an additional washing with saturated aqueous $\mathrm{NaHCO}_{3}$ solution (saturated aqueous $\mathrm{NH}_{4} \mathrm{Cl}$ solutions) was performed. Washing with brine, drying over anhydrous $\mathrm{Na}_{2} \mathrm{SO}_{4}$, and evaporation of the solvent under reduced pressure followed by chromatography on a silica gel column (60-120 mesh) with the indicated eluent furnished the corresponding products. Where solutions were filtered through a Celite pad, the pad was additionally washed with the same solvent used, and the washings were incorporated to the main organic layer. ${ }^{1} \mathrm{H}$ and ${ }^{13} \mathrm{C}$ NMR chemical shifts $(\delta)$ are reported in ppm, and coupling constants $(J)$ are reported in hertz $(\mathrm{Hz})$. High resolution mass spectra were run by the electron impact mode (ESIMS, $70 \mathrm{eV})$ or by the FAB mode $(m$ nitrobenzyl alcohol matrix) using an orbitrap mass analyzer. IR data were measured with oily films on $\mathrm{NaCl}$ plates (oils) or $\mathrm{KBr}$ pellets (solids). Specific optical rotations $[\alpha]^{\mathrm{D}}$ are given in $10^{-1} \mathrm{deg} \mathrm{cm}^{2} \mathrm{~g}^{-1}$ and were measured at $25{ }^{\circ} \mathrm{C}$ or otherwise mentioned. The following abbreviations are used to designate signal multiplicity: $\mathrm{s}=$ singlet, $\mathrm{d}=$ doublet, $\mathrm{t}=$ triplet, $\mathrm{q}=$ quartet, quin $=$ quintet, $\mathrm{m}=$ multiplet, $\mathrm{br}=$ broad.

\section{EXPERIMENTAL SECTION}

\section{(S)-5-((tert-Butyldiphenylsilyl)oxy)methyl)dihydrofuran-2(3H)-one (8):}




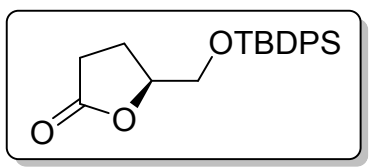

To a stirred solution of $(S)$-5-(hydroxymethyl)dihydrofuran-2(3H)-one $(2.0 \mathrm{~g}, 17.24 \mathrm{mmol})$ in $\mathrm{CH}_{2} \mathrm{Cl}_{2}(60 \mathrm{~mL})$ under nitrogen atmosphere, was added imidazole (2.3 g, $\left.34.48 \mathrm{mmol}\right)$ followed by tert-butyldiphenylchlorosilane $(5.6 \mathrm{~g}, 20.68 \mathrm{mmol})$ at $0{ }^{\circ} \mathrm{C}$ and allowed to stir for $30 \mathrm{~min}$. After completion of the reaction (monitored by TLC), the reaction mixture was quenched with water $(50 \mathrm{~mL})$ and diluted with $\mathrm{CH}_{2} \mathrm{Cl}_{2}(100 \mathrm{~mL})$, the organic layer was separated. The organic layer was washed with brine $(2 \times 50 \mathrm{~mL})$, dried over anhydrous $\mathrm{Na}_{2} \mathrm{SO}_{4}$, concentrated under reduced pressure to obtain the crude product which on purification by silica gel column chromatography purification (ethyl acetate: hexane produced $=1: 19$ ) furnished the desired lactone $8(5.42 \mathrm{~g}, 89 \%)$. $[\alpha]_{\mathrm{D}}{ }^{25}+28.7\left(c 0.9, \mathrm{CHCl}_{3}\right)$; lit. ${ }^{7 \mathrm{~d}}[\alpha]_{\mathrm{D}}+28.95\left(c\right.$ 2.0, $\left.\mathrm{CHCl}_{3}\right)$; IR $(\mathrm{KBr})$ : $v_{\max } 2952,2858,1772,1110,702 \mathrm{~cm}^{-1} ;{ }^{1} \mathrm{H} \mathrm{NMR}\left(500 \mathrm{MHz}, \mathrm{CDCl}_{3}\right): \delta 7.70-7.63(\mathrm{~m}, 4 \mathrm{H})$, $7.47-7.65(\mathrm{~m}, 6 \mathrm{H}), 4.62-4.57(\mathrm{~m}, 1 \mathrm{H}), 3.88(\mathrm{dd}, J=11.44,3.35 \mathrm{~Hz}, 1 \mathrm{H}), 3.69(\mathrm{dd}, J=11.44$, $3.35 \mathrm{~Hz}, 1 \mathrm{H}), 2.71-2.63(\mathrm{~m}, 1 \mathrm{H}), 2.55-2.47(\mathrm{~m}, 1 \mathrm{H}), 2.33-2.18(\mathrm{~m}, 2 \mathrm{H}), 1.06(\mathrm{~s}, 9 \mathrm{H}) \mathrm{ppm}$; ${ }^{13} \mathrm{C}$ NMR $\left(75 \mathrm{MHz}, \mathrm{CDCl}_{3}\right): \delta 77.4,135.6,135.5,129.9,127.8,79.9,65.5,28.5,26.7,23.6,19.2$ ppm; HRMS (ESI): m/z calcd for $\mathrm{C}_{21} \mathrm{H}_{26} \mathrm{O}_{3} \mathrm{Si}[\mathrm{M}+\mathrm{Na}]^{+} 377.1549$, found 377.1568.

\section{(S,E)-Ethyl-7-((tert-butyldiphenylsilyl)oxy)-6-hydroxyhept-2-enoate (10):}

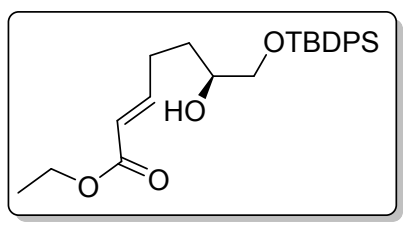

To a stirred solution of lactone $8(4.5 \mathrm{~g}, 12.71 \mathrm{mmol})$ in $\mathrm{CH}_{2} \mathrm{Cl}_{2}(50 \mathrm{~mL})$ at $-78{ }^{\circ} \mathrm{C}$ under nitrogen atmosphere, DIBAL- $H(9.98 \mathrm{~mL}, 1.4 \mathrm{M}$ in toluene, $13.98 \mathrm{mmol})$ was slowly added over a period of $15 \mathrm{~min}$. After $30 \mathrm{~min}$ of stirring at the same temperature (TLC), the reaction was 
quenched by slow addition of saturated sodium potassium tartrate solution $(50 \mathrm{~mL})$, diluted with $\mathrm{CH}_{2} \mathrm{Cl}_{2}(50 \mathrm{~mL})$ and allowed to stir at room temperature for another $2 \mathrm{~h}$ to get a clear two separated layers. The organic layer was separated and the aqueous layer extracted with $\mathrm{CH}_{2} \mathrm{Cl}_{2}$ $(2 \times 80 \mathrm{~mL})$. The combined organic layer was washed with brine $(2 \times 50 \mathrm{~mL})$, dried over anhydrous $\mathrm{Na}_{2} \mathrm{SO}_{4}$, concentrated under reduced pressure to provide crude lactol which was directly used for the next reaction without purification. The corresponding lactol was dissolved in benzene $(100 \mathrm{~mL})$ and was added ethoxycarbonylmethylenetriphenyl phosphorane $(8.84 \mathrm{~g}$, $25.42 \mathrm{mmol}$ ) at room temperature. After stirring for $4 \mathrm{~h}$ at $100{ }^{\circ} \mathrm{C}$, the reaction mixture was concentrated under reduced pressure and purified by silica gel column chromatography (ethyl acetate/hexane $=1: 19)$ to give $9(4.43 \mathrm{~g}, 82 \%$ over two steps $) .[\alpha]_{\mathrm{D}}^{25}-6.07\left(c 1.8, \mathrm{CHCl}_{3}\right)$; IR (KBr): $v_{\max } 3427,2978,1716,1445,1151,1039,922 \mathrm{~cm}^{-1} ;{ }^{1} \mathrm{H}$ NMR (400 MHz, $\left.\mathrm{CDCl}_{3}\right): \delta 7.67$ $-7.63(\mathrm{~m}, 4 \mathrm{H}), 7.45-7.36(\mathrm{~m}, 6 \mathrm{H}), 6.94(\mathrm{dt}, J=13.8,7.0 \mathrm{~Hz}, 1 \mathrm{H}), 5.81(\mathrm{dt}, J=17.2,3.2 \mathrm{~Hz}$, $1 \mathrm{H}), 4.18(\mathrm{q}, J=14.3,7.2 \mathrm{~Hz}, 2 \mathrm{H}), 3.75-3.68(\mathrm{~m}, 1 \mathrm{H}), 3.65(\mathrm{dd}, J=10.2,3.6 \mathrm{~Hz}, 1 \mathrm{H}), 3.49$ $(\mathrm{dd}, J=10.2,7.2 \mathrm{~Hz}, 1 \mathrm{H}), 2.49(\mathrm{~d}, J=3.7 \mathrm{~Hz}, 1 \mathrm{H}), 2.40-2.19(\mathrm{~m}, 2 \mathrm{H}), 1.66-1.46(\mathrm{~m}, 2 \mathrm{H})$, $1.28(\mathrm{t}, J=14.3,7.1 \mathrm{~Hz}, 3 \mathrm{H}), 1.07(\mathrm{~s}, 9 \mathrm{H}) \mathrm{ppm} ;{ }^{13} \mathrm{C} \mathrm{NMR}\left(125 \mathrm{MHz}, \mathrm{CDCl}_{3}\right): \delta 166.6,148.5$, $135.5,133.0,130.0,127.8,121.6,71.0,67.8,60.2,31.1,28.2,26.8,19.2,14.2$ ppm; HRMS (ESI): $m / z$ calcd. for $\mathrm{C}_{25} \mathrm{H}_{34} \mathrm{O}_{4} \mathrm{Si}[\mathrm{M}+\mathrm{Na}]^{+}:$449.2119, found 449.2134.

\section{(S,E)-Ethyl-7-((tert-butyldiphenylsilyl)oxy)-6-((methylsulfonyl)oxy)hept-2-enoate (11):}

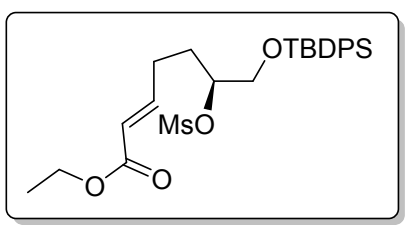

To the mixture of $9(4.1 \mathrm{~g}, 9.61 \mathrm{mmol})$ and triethylamine $(2.7 \mathrm{~mL}, 19.23 \mathrm{mmol})$ in $\mathrm{CH}_{2} \mathrm{Cl}_{2}(60$ $\mathrm{mL})$, methanesulphonylchloride $(1.1 \mathrm{~mL}, 14.42 \mathrm{mmol})$ was added dropwise at $0{ }^{\circ} \mathrm{C}$. After stirring for $3 \mathrm{~h}$ at same temperature, the reaction was quenched with saturated aqueous $\mathrm{NaHCO}_{3}$ 
$(30 \mathrm{~mL})$. The organic layer was separated and the aqueous layer was extracted with $\mathrm{CH}_{2} \mathrm{Cl}_{2}(2 \times$ $50 \mathrm{~mL})$. The combined organic layer was washed with brine $(70 \mathrm{~mL})$, dried over anhydrous $\mathrm{Na}_{2} \mathrm{SO}_{4}$, concentrated under reduced pressure to obtain the crude product which was passed through short plug silica gel to get the mesylated product $10(4.59 \mathrm{~g}, 95 \%)$ as a colorless oil. $[\alpha]_{\mathrm{D}}{ }^{25}-4.49\left(c 1.95, \mathrm{CHCl}_{3}\right)$; IR (KBr): $v_{\max } 2939,2861,1715,1353,1173,701 \mathrm{~cm}^{-1} ;{ }^{1} \mathrm{H} \mathrm{NMR}$ $\left(400 \mathrm{MHz}, \mathrm{CDCl}_{3}\right): \delta 7.74-7.62(\mathrm{~m}, 4 \mathrm{H}), 7.49-7.35(\mathrm{~m}, 6 \mathrm{H}), 6.90(\mathrm{dt}, J=15.65,13.69,6.84$ $\mathrm{Hz}, 1 \mathrm{H}), 5.83(\mathrm{dt}, J=15.65 \mathrm{~Hz}, 1 \mathrm{H}), 4.76-4.69(\mathrm{~m}, 1 \mathrm{H}), 4.18(\mathrm{q}, J=14.18,7.09 \mathrm{~Hz}, 2 \mathrm{H}), 3.85$ $-3.71(\mathrm{~m}, 2 \mathrm{H}), 2.96(\mathrm{~s}, 3 \mathrm{H}), 2.38-2.21(\mathrm{~m}, 2 \mathrm{H}), 1.93-1.79(\mathrm{~m}, 2 \mathrm{H}), 1.28(\mathrm{t}, J=14.30,7.09$ $\mathrm{Hz}, 2 \mathrm{H}), 1.07$ (s, 9H) ppm; ${ }^{13} \mathrm{C} \mathrm{NMR}\left(100 \mathrm{MHz}, \mathrm{CDCl}_{3}\right): \delta 166.3,146.9,135.5,135.4,134.7$, $132.6,132.5,130.0,130.0,127.9,122.2,82.3,65.1,60.2,38.6,29.7,27.4,26.8,19.2,14.2 \mathrm{ppm}$ HRMS (ESI): $m / z$ calcd. for $\mathrm{C}_{26} \mathrm{H}_{36} \mathrm{O}_{6} \mathrm{SSi}[\mathrm{M}+\mathrm{Na}]^{+}: 527.1894$, found 527.1902 .

(S)-Ethyl-2-((2R,5R)-5-(((tert-butyldiphenylsilyl)oxy)methyl)tetrahydrofuran-2-yl)-2hydroxyacetate (11):

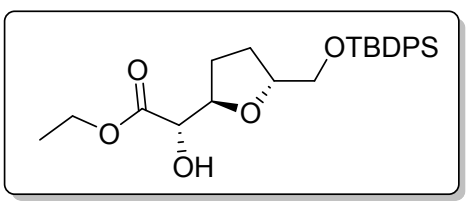

A mixture of $\mathrm{K}_{2} \mathrm{OsO}_{2}(\mathrm{OH})_{2}$ (16.6 mg, $0.045 \mathrm{mmol}, 0.006$ equiv), (DHQD) ${ }_{2} \mathrm{PHAL}$ ( $88 \mathrm{mg}, 0.11$ mmol, 0.015 equiv.), $\mathrm{K}_{3} \mathrm{Fe}(\mathrm{CN})_{6}\left(7.44 \mathrm{~g}, 22.6 \mathrm{mmol}, 3.0\right.$ equiv), $\mathrm{K}_{2} \mathrm{CO}_{3}(3.12 \mathrm{~g}, 22.6$ mmol, 3.0 equiv) and $\mathrm{MeSO}_{2} \mathrm{NH}_{2}\left(716 \mathrm{mg}, 7.53 \mathrm{mmol}, 1.0\right.$ equiv) in 1:1 $t-\mathrm{BuOH} / \mathrm{H}_{2} \mathrm{O}(100 \mathrm{~mL})$ was stirred for 30 minutes at room temperature. This mixture was slowly poured into $4{ }^{\circ} \mathrm{C}$ solution of $\mathbf{1 0}$ (3.8 g, $7.53 \mathrm{mmol}, 1.0$ equiv) in $t-\mathrm{BuOH} / \mathrm{H}_{2} \mathrm{O}(500 \mathrm{~mL})$. The reaction was stirred at $4{ }^{\circ} \mathrm{C}$ for 24 h. After completion of the reaction, solid $\mathrm{Na}_{2} \mathrm{~S}_{2} \mathrm{O}_{3}(10 \mathrm{~g})$ was added and the mixture was stirred for $1.5 \mathrm{~h}$ while warming to room temperature (color change from orange to dark green). After phase separation, the aqueous layer was extracted with ethyl acetate $(5 \times 20 \mathrm{~mL})$. The combined 
organic layers were dried over $\mathrm{Na}_{2} \mathrm{SO}_{4}$, concentrated under reduced pressure to give crude product which was purified by silica gel column chromatography (ethyl acetate/hexane $=4: 1$ ) to give $11(2.93 \mathrm{~g}, 88 \%) \cdot[\alpha]_{\mathrm{D}}^{25}-8.3\left(c 1.6, \mathrm{CHCl}_{3}\right)$; IR (KBr): $v_{\max } 3477,2931,2858,1739,1110$, $704 \mathrm{~cm}^{-1} ;{ }^{1} \mathrm{H}$ NMR (400 MHz, $\left.\mathrm{CDCl}_{3}\right): \delta 7.73-7.60(\mathrm{~m}, 4 \mathrm{H}), 7.46-7.34(\mathrm{~m}, 6 \mathrm{H}), 4.37(\mathrm{dt}, J$ $=2.20,7.09,13.93 \mathrm{~Hz}, 1 \mathrm{H}), 4.43-4.16(\mathrm{~m}, 3 \mathrm{H}), 4.07(\mathrm{dd}, J=1.95,8.06 \mathrm{~Hz}, 1 \mathrm{H}), 3.63(\mathrm{dq}, J=$ 4.64, 10.63, 25.30, 2H), $2.94(\mathrm{~d}, J=8.18,1 \mathrm{H}), 2.13-2.20(\mathrm{~m}, 3 \mathrm{H}), 1.98-1.86(\mathrm{~m}, 1 \mathrm{H}), 1.27(\mathrm{t}$, $J=7.21,14.30 \mathrm{~Hz}, 3 \mathrm{H}), 1.04(\mathrm{~s}, 9 \mathrm{H}) \mathrm{ppm} ;{ }^{13} \mathrm{C} \mathrm{NMR}\left(125 \mathrm{MHz}, \mathrm{CDCl}_{3}\right): \delta 173.0,135.6,135.5$, $134.8,133.6,133.5,129.6,129.5,127.6,127.6,80.8,80.0,72.5,66.2,61.6,28.1,27.8,26.7$, 19.2, 14.1 ppm; HRMS (ESI) : $m / z$ calcd. for $\mathrm{C}_{25} \mathrm{H}_{34} \mathrm{O}_{5} \mathrm{Si}[\mathrm{M}+\mathrm{Na}]^{+}:$465.2068, found 465.2085.

\section{(R)-1-((2R,5R)-5-(((tert-Butyldiphenylsilyl)oxy)methyl)tetrahydrofuran-2-yl)ethane-1,2-} diol (12):

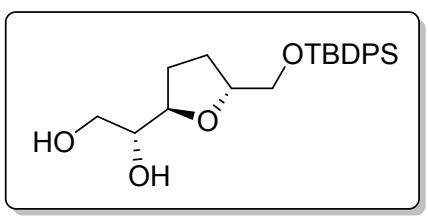

To a solution of ester $11(2.5 \mathrm{~g}, 5.65 \mathrm{mmol})$ in diethyl ether $(50 \mathrm{~mL}), \mathrm{LiBH}_{4}(184 \mathrm{mg}, 8.48$ mmol) was added at $0{ }^{\circ} \mathrm{C}$ in a single portion. $\mathrm{MeOH}(5 \mathrm{~mL})$ was added to the above reaction mixture at same temperature. The above reaction mixture was stirred for additional $2 \mathrm{~h}$ at room temperature. After completion of reaction (TLC), it was quenched with aqueous $\mathrm{NaHCO}_{3}$ solution $(20 \mathrm{~mL})$ and extracted with ethyl acetate $(3 \times 50 \mathrm{~mL})$, the combined organic layers were dried over $\mathrm{Na}_{2} \mathrm{SO}_{4}$, evaporated under reduced pressure to crude product which was purified by silica gel column chromatography (ethyl acetate/hexane =1:1) to furnish diol $12(1.96 \mathrm{~g}, 87 \%)$ as a colorless viscous liquid $[\alpha]_{\mathrm{D}}{ }^{25}-4.0\left(c\right.$ 1.3, $\left.\mathrm{CHCl}_{3}\right)$; IR $(\mathrm{KBr}): v_{\max } 3421,2931,2858,1427$, 1110, $703 \mathrm{~cm}^{-1} ;{ }^{1} \mathrm{H}$ NMR $\left(400 \mathrm{MHz}, \mathrm{CDCl}_{3}\right): \delta 7.70-7.66(\mathrm{~m}, 4 \mathrm{H}), 7.45-7.35(\mathrm{~m}, 6 \mathrm{H}), 4.18-$ $4.09(\mathrm{~m}, 1 \mathrm{H}), 4.03,3.97(\mathrm{~m}, 1 \mathrm{H}), 3.72-3.60(\mathrm{~m}, 4 \mathrm{H}), 3.53(\mathrm{q}, J=4.40,8.68 \mathrm{~Hz}, 1 \mathrm{H}), 2.72(\mathrm{br}$, 
1H), 2.58 (br, 1H), $2.03-1.76(\mathrm{~m}, 4 \mathrm{H}), 1.05(\mathrm{~s}, 9 \mathrm{H}) \mathrm{ppm} ;{ }^{13} \mathrm{C} \mathrm{NMR}\left(100 \mathrm{MHz}, \mathrm{CDCl}_{3}\right): \delta 135.6$, 133.6, 133.5, 129.6, 127.6, 80.7, 80.2, 72.8, 66.3, 64.9, 28.1, 27.9, 26.8, 19.2 ppm; HRMS (ESI): $m / z$ calcd. for $\mathrm{C}_{23} \mathrm{H}_{32} \mathrm{O}_{4} \mathrm{Si}[\mathrm{M}+\mathrm{Na}]^{+}$: 423.1962, found 423.1970 .

(2R,5R)-5-(((tert-Butyldiphenylsilyl)oxy)methyl)tetrahydrofuran-2-carbaldehyde (13):

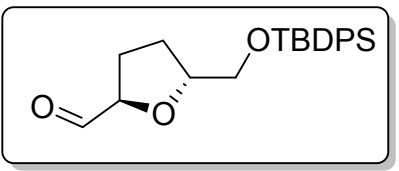

To the solution of $12(1.4 \mathrm{~g}, 3.5 \mathrm{mmol})$ in $\mathrm{CH}_{2} \mathrm{Cl}_{2}(80 \mathrm{~mL}), \mathrm{NaIO}_{4}$-silica $(35 \mathrm{~g})$ was added at 0 ${ }^{\circ} \mathrm{C}$. The reaction was stirred for $30 \mathrm{~min}$ at room temperature. After completion of reaction (TLC), the reaction mixture was filtered and washed with $\mathrm{CH}_{2} \mathrm{Cl}_{2}(2 \times 30 \mathrm{~mL})$. The resulting solution was concentrated under reduced pressure to provide the crude product which upon silica-gel column chromatography purification (ethyl acetate/hexane $=1: 5)$ afforded $\mathbf{1 3}(0.828 \mathrm{~g}, 90 \%)$ as a colorless viscous liquid. $[\alpha]_{\mathrm{D}}{ }^{25}+3.41\left(c 3.45, \mathrm{CHCl}_{3}\right)$; IR $(\mathrm{KBr}): v_{\max } 2931,2858,1733,1427$, 1110, $704 \mathrm{~cm}^{-1} ;{ }^{1} \mathrm{H}$ NMR (500 MHz, $\left.\mathrm{CDCl}_{3}\right): \delta 9.66(\mathrm{~d}, J=1.67 \mathrm{~Hz}, 1 \mathrm{H}), 7.70-7.66(\mathrm{~m}, 4 \mathrm{H})$, $7.44-7.35(\mathrm{~m}, 6 \mathrm{H}), 4.36-4.32(\mathrm{~m}, 1 \mathrm{H}), 4.29-4.22(\mathrm{~m}, 1 \mathrm{H}), 3.71(\mathrm{dq}, J=25.63,10.83,4.42$ $\mathrm{Hz}, 2 \mathrm{H}), 2.24-2.16(\mathrm{~m}, 1 \mathrm{H}), 2.01-1.90(\mathrm{~m}, 3 \mathrm{H}), 1.06(\mathrm{~s}, 9 \mathrm{H}) \mathrm{ppm} ;{ }^{13} \mathrm{C} \mathrm{NMR}(125 \mathrm{MHz}$ $\left.\mathrm{CDCl}_{3}\right): \delta 202.8,135.5,133.3,133.3,129.7,127.6,83.3,81.0,65.9,27.3,27.2,26.8,19.2$ ppm; HRMS (ESI): $m / z$ calcd. for $\mathrm{C}_{22} \mathrm{H}_{28} \mathrm{O}_{3} \mathrm{Si}\left[\mathrm{M}+\mathrm{NH}_{4}\right]^{+}: 386.2146$, found 386.2155.

(E)-Ethyl-3-((2R,5R)-5-(((tert-butyldiphenylsilyl)oxy)methyl)tetrahydrofuran-2-yl)acrylate (14):

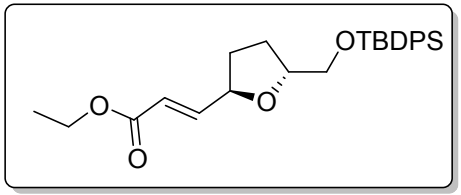


To a stirred suspension of ethyl 2-(triphenylposphoranylidene)acetate $(1.5 \mathrm{~g}, 4.34 \mathrm{mmol})$ in toluene $(50 \mathrm{~mL})$ was added a solution of $\mathbf{1 3}(800 \mathrm{mg}, 2.17 \mathrm{mmol})$ was added at reflux and maintained for $5 \mathrm{~h}$. Cool the reaction mass to room temperature and poured into water carefully. The mixture was extracted with ethyl acetate $(2 \times 40 \mathrm{~mL})$ and the organic layer was dried over anhydrous $\mathrm{Na}_{2} \mathrm{SO}_{4}$ and evaporated under reduced pressure. The resulting syrup was purified by silica gel column chromatography $(\mathrm{EtOAc} / \mathrm{hexane}=1: 9)$ to give compound $15(846 \mathrm{mg}, 89 \%)$ as a colorless liquid. $[\alpha]_{\mathrm{D}}{ }^{25}+6.2\left(c 2.2, \mathrm{CHCl}_{3}\right)$; IR (KBr): $v_{\max } 2932,2859,1721,1187,1110,704$ $\mathrm{cm}^{-1} ;{ }^{1} \mathrm{H}$ NMR $\left(400 \mathrm{MHz}, \mathrm{CDCl}_{3}\right): \delta 7.73-7.57(\mathrm{~m}, 4 \mathrm{H}), 7.45-7.32(\mathrm{~m}, 6 \mathrm{H}), 6.91(\mathrm{dd}, J=$ 15.65, $4.86 \mathrm{~Hz}, 1 \mathrm{H}), 6.02(\mathrm{dd}, J=15.65,1.58 \mathrm{~Hz}, 1 \mathrm{H}), 4.63-4.57(\mathrm{~m}, 1 \mathrm{H}), 4.30-4.09(\mathrm{~m}, 1 \mathrm{H})$, $4.20(\mathrm{q}, J=14.30,7.21 \mathrm{~Hz}, 2 \mathrm{H}), 3.67(\mathrm{~d}, J=4.64 \mathrm{~Hz}, 2 \mathrm{H}), 2.23-1.58(\mathrm{~m}, 3 \mathrm{H}), 1.29(\mathrm{t}, J=$ 14.30, $7.21 \mathrm{~Hz}, 3 \mathrm{H}), 1.36-1.20(\mathrm{~m}, 2 \mathrm{H}), 1.06(\mathrm{~s}, 9 \mathrm{H}) \mathrm{ppm} ;{ }^{13} \mathrm{C} \mathrm{NMR}\left(100 \mathrm{MHz}, \mathrm{CDCl}_{3}\right): \delta$ $166.6,148.5,135.6,133.5,129.6,127.8,127.6,120.1,79.8,78.0,66.3,60.3,31.8,27.6,26.8$, 19.2, 14.2 ppm; HRMS (ESI): $m / z$ calcd. for $\mathrm{C}_{26} \mathrm{H}_{34} \mathrm{O}_{4} \mathrm{Si}\left[\mathrm{M}+\mathrm{NH}_{4}\right]^{+}: 456.2564$ found 456.2564 .

\section{Ethyl-3-((2R,5R)-5-(((tert-butyldiphenylsilyl)oxy)methyl)tetrahydrofuran-2-yl)propanoate} (15):

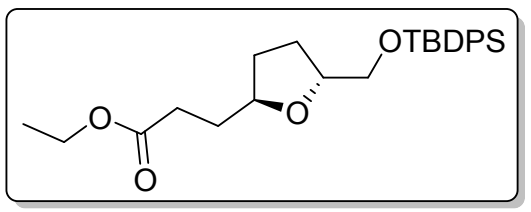

To a solution of $\alpha, \beta$-unsaturated ester $14(800 \mathrm{mg}, 1.82 \mathrm{mmol})$ in ethyl acetate $(15 \mathrm{~mL})$, was added $\mathrm{Pd} / \mathrm{C}(0.2 \mathrm{~g}, 0.18 \mathrm{mmol})$ under hydrogen atmosphere at room temperature and stirring continued for $2 \mathrm{~h}$. After completion of the reaction (monitored by TLC), the reaction mass was passed through a small Celite pad and washed with ethyl acetate $(2 \times 25 \mathrm{~mL})$. The filtrate was concentrated under reduced pressure to provide crude product which was purified by silica gel column chromatography (ethyl acetate/hexane $=1: 19)$ to furnish $15(794 \mathrm{mg}, 99 \%)$ as a colorless 
viscous liquid. $[\alpha]_{\mathrm{D}}{ }^{25}-3.9\left(c 2.2, \mathrm{CHCl}_{3}\right)$; IR (KBr): $v_{\max } 2930,2858,1732,1108,701 \mathrm{~cm}^{-1} ;{ }^{1} \mathrm{H}$ $\operatorname{NMR}\left(500 \mathrm{MHz}, \mathrm{CDCl}_{3}\right): \delta 7.71-7.66(\mathrm{~m}, 4 \mathrm{H}), 7.43-7.35(\mathrm{~m}, 6 \mathrm{H}), 4.14-4.08(\mathrm{~m}, 3 \mathrm{H}), 3.99$ $-3.93(\mathrm{~m}, 1 \mathrm{H}), 3.63(\mathrm{dd}, J=4.57,1.52 \mathrm{~Hz}, 2 \mathrm{H}), 2.49-2.42(\mathrm{~m}, 1 \mathrm{H}), 2.40-2.33(\mathrm{~m}, 1 \mathrm{H}), 2.06$ $-1.96(\mathrm{~m}, 2 \mathrm{H}), 1.88-1.78(\mathrm{~m}, 3 \mathrm{H}), 1.56-1.47(\mathrm{~m}, 1 \mathrm{H}), 1.64(\mathrm{br} \mathrm{s}, 1 \mathrm{H}), 1.24(\mathrm{t}, \quad J=14.19$, $7.17 \mathrm{~Hz}, 3 \mathrm{H}), 1.05$ (s, 9H) ppm; ${ }^{13} \mathrm{C}$ NMR (100 MHz, $\left.\mathrm{CDCl}_{3}\right): \delta 173.6,135.6,133.7,129.5$, 127.6, 78.9, 78.5, 66.5, 60.3, 31.7, 31.2, 30.8, 29.7, 28, 26.8, 19.2, 14.2 ppm; HRMS (ESI): $m / z$ calcd for $m / z$ calcd. for $\mathrm{C}_{26} \mathrm{H}_{36} \mathrm{O}_{4} \mathrm{Si}[\mathrm{M}+\mathrm{Na}]^{+} 463.2281$, found 463.2267 .

\section{Dimethyl-4-((2R,5R)-5-((tert-butyldiphenylsilyloxy)methyl)tetrahydrofuran-2-yl)-2-} oxobutylphosphonate (5):

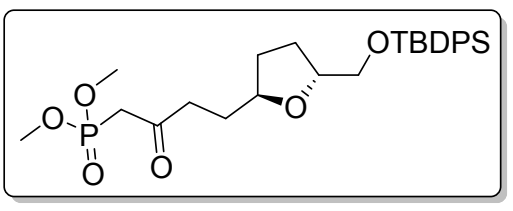

To a stirred solution of the dimethylmethylphosphonate $(570 \mathrm{mg}, 4.60 \mathrm{mmol})$ in THF $(30 \mathrm{~mL})$, $n$-BuLi (1.7 mL, $4.29 \mathrm{mmol}, 2.5 \mathrm{M}$ in hexane) was slowly added at $-78{ }^{\circ} \mathrm{C}$ under Argon atmosphere and allowed to slowly warm to $0{ }^{\circ} \mathrm{C}$. After $1 \mathrm{~h}$, the reaction mixture was again cooled to $-78{ }^{\circ} \mathrm{C}$ and the solution of ester $15(450 \mathrm{mg}, 1.02 \mathrm{mmol})$ in THF $(30 \mathrm{~mL})$ was slowly added and stirred at the same temperature for $1 \mathrm{~h}$. After complete consumption of the starting material (monitored by TLC), the reaction mixture was quenched with saturated $\mathrm{NH}_{4} \mathrm{Cl}(20 \mathrm{~mL})$. The organic layer was separated and the aqueous layer was extracted with ethyl acetate $(2 \times 40$ $\mathrm{mL})$. The combined organic layers were washed with brine $(50 \mathrm{~mL})$ and dried over $\mathrm{Na}_{2} \mathrm{SO}_{4}$. The organic layer was concentrated under reduced pressure to obtain the crude mass which on purification by silica gel column chromatography (ethyl acetate/hexane $=1: 1$ ) afforded the desired $6(428 \mathrm{mg}, 90 \%)$ as a colorless liquid. $[\alpha]_{\mathrm{D}}{ }^{25}-4.0\left(c 1.0, \mathrm{CHCl}_{3}\right)$; IR $(\mathrm{KBr}): v_{\max } 2933$, 2859, 1716, 1109, 1034, $702 \mathrm{~cm}^{-1 ;}{ }^{1} \mathrm{H}$ NMR $\left(500 \mathrm{MHz}, \mathrm{CDCl}_{3}\right): \delta 7.70-7.65(\mathrm{~m}, 4 \mathrm{H}), 7.44-$ 
$7.35(\mathrm{~m}, 6 \mathrm{H}), 4.12-4.05(\mathrm{~m}, 1 \mathrm{H}), 3.96-3.89(\mathrm{~m}, 1 \mathrm{H}), 3.78(\mathrm{~s}, 3 \mathrm{H}), 3.76(\mathrm{~s}, 3 \mathrm{H}), 3.65-3.58$ $(\mathrm{m}, 2 \mathrm{H}), 3.16-3.04(\mathrm{~m}, 2 \mathrm{H}), 2.80-2.61(\mathrm{~m}, 2 \mathrm{H}), 2.05-1.95(\mathrm{~m}, 2 \mathrm{H}), 1.85-1.70(\mathrm{~m}, 3 \mathrm{H})$ $1.55-1.46(\mathrm{~m}, 1 \mathrm{H}), 1.05(\mathrm{~s}, 9 \mathrm{H}) \mathrm{ppm} ;{ }^{13} \mathrm{C} \mathrm{NMR}\left(100 \mathrm{MHz}, \mathrm{CDCl}_{3}\right): \delta 201.7,201.7,135.6$, $133.7,129.5,127.6,78.9,78.3,66.5,53.0,52.9,41.8,40.9,40.7,31.7,29.5,28,26.8,19.2$ ppm; HRMS (ESI): $m / z$ calcd. for $\mathrm{C}_{27} \mathrm{H}_{39} \mathrm{O}_{6} \mathrm{Psi}\left[\mathrm{M}+\mathrm{NH}_{4}\right]^{+}: 536.2591$ found 536.2598 .

\section{(2R,3S)-7-((4-Methoxybenzyl)oxy)-2-methylheptane-1,3-diol (17):}

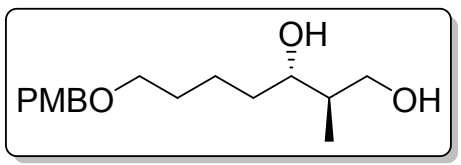

To a solution of chiral epoxide 16 (1.8 g, $6.76 \mathrm{mmol})$ in dry THF $(40 \mathrm{~mL}), \mathrm{CuI}(0.128 \mathrm{~g}, 0.676$ mmol) was added and the mixture was stirred at $25{ }^{\circ} \mathrm{C}$ for $30 \mathrm{~min}$. It was cooled to $-5{ }^{\circ} \mathrm{C}$ and methyl magnesium bromide $(20.28 \mathrm{~mL}, 1 \mathrm{M}$ in THF, $20.28 \mathrm{mmol})$ was slowly added at the same temperature. It was allowed to stir for another $12 \mathrm{~h}$ at same temperature. After completion of the reaction (TLC), it was quenched with saturated $\mathrm{NH}_{4} \mathrm{Cl}(30 \mathrm{~mL})$ and diluted with ethyl acetate (50 $\mathrm{mL})$. The two layers were separated and the aqueous layer was extracted with ethyl acetate $(2 \times$ $50 \mathrm{~mL})$. The combined organic layers were washed with brine $(100 \mathrm{~mL})$, dried over anhydrous $\mathrm{Na}_{2} \mathrm{SO}_{4}$ and concentrated under reduced pressure to obtain the crude product which was then purified by column chromatography over silica gel (ethyl acetate/hexane $=1: 1$ ) to afford the product $17(1.43 \mathrm{~g}, 75 \%)$. $[\alpha]_{\mathrm{D}}{ }^{25}-8.0\left(c 0.51, \mathrm{CHCl}_{3}\right)$; IR (KBr): $v_{\max } 3385,2936,2863,1512$, 1247, 1032, $770 \mathrm{~cm}^{-1} ;{ }^{1} \mathrm{H}$ NMR (500 MHz, $\left.\mathrm{CDCl}_{3}\right): \delta 7.25(\mathrm{~d}, J=8.54 \mathrm{~Hz}, 2 \mathrm{H}), 6.87(\mathrm{~d}, J=$ $8.69 \mathrm{~Hz}, 2 \mathrm{H}), 4.42(\mathrm{~s}, 2 \mathrm{H}), 3.79(\mathrm{~s}, 3 \mathrm{H}), 3.71(\mathrm{dd}, J=10.83,3.66 \mathrm{~Hz}, 1 \mathrm{H}), 3.59-3.49(\mathrm{~m}, 2 \mathrm{H})$, $3.49-3.42(\mathrm{~m}, 2 \mathrm{H}), 1.73-1.36(\mathrm{~m}, 7 \mathrm{H}), 0.85(\mathrm{~d}, J=7.01 \mathrm{~Hz}, 3 \mathrm{H}) \mathrm{ppm} ;{ }^{13} \mathrm{C}$ NMR $(125 \mathrm{MHz}$ $\left.\mathrm{CDCl}_{3}\right): \delta 159.1,132.0,129.3,113.7,76.8,72.5,70.0,67.5,55.2,39.7,34.8,29.5,21.8,13.8$ ppm; HRMS (ESI): $m / z$ calcd. for $\mathrm{C}_{16} \mathrm{H}_{26} \mathrm{O}_{4}[\mathrm{M}+\mathrm{Na}]^{+}: 305.1723$, found 305.1739. 
(5S,6R)-5-(4-((4-Methoxybenzyl)oxy)butyl)-2,2,3,3,6,9,9,10,10-nonamethyl-4,8-dioxa-3,9disilaundecane (18):

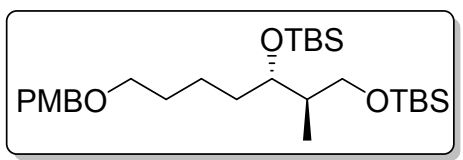

To a stirred solution of $17(1.2 \mathrm{~g}, 4.25 \mathrm{mmol})$ in $\mathrm{CH}_{2} \mathrm{Cl}_{2}(40 \mathrm{~mL})$ under nitrogen atmosphere, was added DIPEA $(2.67 \mathrm{~mL}, 14.88 \mathrm{mmol})$ followed by tert-butyldimethylsilyl trifluoromethanesulfonate $(2.81 \mathrm{~g}, 10.63 \mathrm{mmol})$ at $0{ }^{\circ} \mathrm{C}$ and was stirred for $6 \mathrm{~h}$. After completion of the reaction (monitored by TLC), it was quenched with water $(20 \mathrm{~mL})$. The organic layer was separated and the aqueous layer was extracted with $\mathrm{CH}_{2} \mathrm{Cl}_{2}(2 \times 40 \mathrm{~mL})$. The combined organic layer was washed with brine $(70 \mathrm{~mL})$, dried over anhydrous $\mathrm{Na}_{2} \mathrm{SO}_{4}$, concentrated under reduced pressure to obtain the crude product which on purification by silica gel column chromatography (ethyl acetate/hexane $=1: 19)$ furnished $18(1.97 \mathrm{~g}, 91 \%) .[\alpha]_{\mathrm{D}}{ }^{25}-6.9\left(c 1.6, \mathrm{CHCl}_{3}\right)$; IR $(\mathrm{KBr})$ : $v_{\max } 2934,2857,1515,1250,1094,837,772 \mathrm{~cm}^{-1} ;{ }^{1} \mathrm{H} \mathrm{NMR}\left(500 \mathrm{MHz}, \mathrm{CDCl}_{3}\right): \delta 7.26(\mathrm{~d}, J=$ $8.68 \mathrm{~Hz}, 2 \mathrm{H}), 6.87(\mathrm{~d}, J=8.80 \mathrm{~Hz}, 2 \mathrm{H}), 4.43(\mathrm{~s}, 2 \mathrm{H}), 3.80(\mathrm{~s}, 3 \mathrm{H}), 3.69(\mathrm{q}, J=9.53,4.64 \mathrm{~Hz}$, 1H), $3.55(\mathrm{dd}, J=10.02,6.48 \mathrm{~Hz}, 1 \mathrm{H}), 3.46-3.37(\mathrm{~m}, 3 \mathrm{H}), 1.88-1.75(\mathrm{~m}, 1 \mathrm{H}), 1.64-1.24$ $(\mathrm{m}, 6 \mathrm{H}), 0.89(\mathrm{~s}, 9 \mathrm{H}), 0.88(\mathrm{~s}, 9 \mathrm{H}), 0.83(\mathrm{~d}, J=6.96 \mathrm{~Hz}, 3 \mathrm{H}), 0.03(\mathrm{~s}, 12 \mathrm{H}) \mathrm{ppm} ;{ }^{13} \mathrm{C}$ NMR $(125$ $\left.\mathrm{MHz}, \mathrm{CDCl}_{3}\right): \delta 159.0,130.8,129.2,113.7,73.1,72.5,70.2,65.2,55.3,40.9,32.4,30.0,25.9$, $21.8,18.3,18.1,12.2,-4.4,-4.6,-5.4,-5.5$ ppm; HRMS (ESI) : $m / z$ calcd. for $\mathrm{C}_{28} \mathrm{H}_{54} \mathrm{O}_{4} \mathrm{Si}_{2}[\mathrm{M}+$ $\left.\mathrm{NH}_{4}\right]^{+}:$528.3898, found 528.3902. 


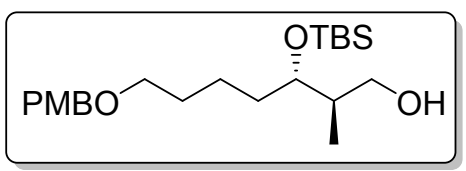

To a stirred solution of $18(1.6 \mathrm{~g}, 3.13 \mathrm{mmol})$ in $\mathrm{CH}_{2} \mathrm{Cl}_{2}(40 \mathrm{~mL})$ and $\mathrm{MeOH}(40 \mathrm{~mL})$, pyridinium $p$-toluenesulfonate $(78 \mathrm{mg}, 0.31 \mathrm{mmol})$ was added at $0{ }^{\circ} \mathrm{C}$. The reaction was stirred for $6 \mathrm{~h}$ at same temperature. Triethylamine $(3 \mathrm{~mL})$ was added to the reaction mixture and concentrated under reduced pressure to get the crude product which was purified by silica gel chromatography (ethyl acetate/hexane $=1: 9)$ to furnish $19(1.1 \mathrm{~g}, 90 \%)$ as a colorless liquid. $[\alpha]_{\mathrm{D}}{ }^{25}+5.3\left(c 0.51 \mathrm{CHCl}_{3}\right)$; IR (KBr): $v_{\max } 3447,2932,2857,1513,1249,772 \mathrm{~cm}^{-1 ;}{ }^{1} \mathrm{H} \mathrm{NMR}$ $\left(500 \mathrm{MHz}, \mathrm{CDCl}_{3}\right): \delta 7.25(\mathrm{~d}, J=8.69 \mathrm{~Hz}, 2 \mathrm{H}), 6.87(\mathrm{~d}, J=8.69 \mathrm{~Hz}, 2 \mathrm{H}), 4.43(\mathrm{~s}, 2 \mathrm{H}), 3.80(\mathrm{~s}$, 3H), $3.77(\mathrm{dd}, J=10.83,3.81 \mathrm{~Hz}, 1 \mathrm{H}), 3.69(\mathrm{dd}, J=11.13,5.18 \mathrm{~Hz}, 1 \mathrm{H}), 3.52(\mathrm{dd}, J=10.83$, $5.18 \mathrm{~Hz}, 1 \mathrm{H}), 3.44(\mathrm{dt}, J=12.97,6.40,1.67 \mathrm{~Hz}, 2 \mathrm{H}), 1.76(\mathrm{~m}, 1 \mathrm{H}), 1.63-1.50(\mathrm{~m}, 4 \mathrm{H}), 1.37$ (q, $J=15.71,7.93 \mathrm{~Hz}, 2 \mathrm{H}), 0.99(\mathrm{~d}, J=7.01 \mathrm{~Hz}, 3 \mathrm{H}), 0.89(\mathrm{~s}, 9 \mathrm{H}), 0.08(\mathrm{~s}, 3 \mathrm{H}), 0.07$ (s, 3H) ppm; ${ }^{13} \mathrm{C} \mathrm{NMR}\left(75 \mathrm{MHz}, \mathrm{CDCl}_{3}\right): \delta 159.1,130.6,129.2,113.7,77.2,72.6,69.9,65.3,55.2,37.7,34.6$, 29.9, 25.8, 21.6, 18.0, 14.6, -4.3, -4.8 ppm; HRMS (ESI): $m / z$ calcd for $\mathrm{C}_{22} \mathrm{H}_{40} \mathrm{O}_{4} \mathrm{Si}[\mathrm{M}+\mathrm{Na}]^{+}$: 419.2594, found 419.2581.

\section{(2S,3S)-3-(tert-Butyldimethylsilyloxy)-7-(4-methoxybenzyloxy)-2-methylheptanal (6):}

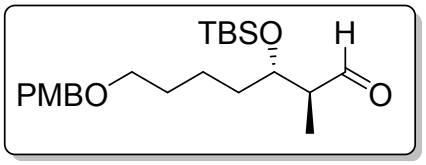

To a solution of alcohol $20(0.9 \mathrm{~g}, 2.27 \mathrm{mmol})$ in $\mathrm{CH}_{2} \mathrm{Cl}_{2}(25 \mathrm{~mL}), \mathrm{NaHCO}_{3}(0.381 \mathrm{~g}, 4.54 \mathrm{~mL})$ and Dess-Martin periodinane $(1.92 \mathrm{~g}, 4.54 \mathrm{mmol})$ were added at $0{ }^{\circ} \mathrm{C}$ under nitrogen atmosphere and was stirred for $2 \mathrm{~h}$ at room temperature. After complete conversion of the starting material (monitored by TLC), the reaction was quenched with water $(20 \mathrm{~mL})$ and diluted with $\mathrm{CH}_{2} \mathrm{Cl}_{2}(25$ $\mathrm{mL})$. The two layers were separated and the aqueous layer was extracted with $\mathrm{CH}_{2} \mathrm{Cl}_{2}(2 \times 30$ 
$\mathrm{mL})$. The combined organic layer was washed with brine $(50 \mathrm{~mL})$, dried over anhydrous $\mathrm{Na}_{2} \mathrm{SO}_{4}$ and concentrated under reduced pressure to get the crude product which was passed through silica gel (ethyl acetate/hexane $=1: 5)$ to afford the aldehyde $7(0.797 \mathrm{~g}, 89 \%)$ as a colorless liquid. $[\alpha]_{\mathrm{D}}^{25}+8.0\left(c\right.$ 1.5, $\left.\mathrm{CHCl}_{3}\right)$; IR $(\mathrm{KBr}): v_{\max } 2930,2856,1724,1512,1250,773 \mathrm{~cm}^{-1} ;{ }^{1} \mathrm{H}$ $\operatorname{NMR}\left(500 \mathrm{MHz}, \mathrm{CDCl}_{3}\right): \delta 9.81(\mathrm{~d}, J=2.59 \mathrm{~Hz}, 1 \mathrm{H}), 5.90(\mathrm{dd}, J=10.83,17.39 \mathrm{~Hz}, 1 \mathrm{H}), 5.20$ (dd, $J=1.52,17.24 \mathrm{~Hz}, 1 \mathrm{H}), 5.00(\mathrm{dd}, J=1.52,10.68 \mathrm{~Hz}, 1 \mathrm{H}), 4.17$ (ddd, $J=6.10,8.24,14.34$ $\mathrm{Hz}, 1 \mathrm{H}), 2.54-2.47(\mathrm{~m}, 1 \mathrm{H}), 1.97-1.88(\mathrm{~m}, 2 \mathrm{H}), 1.83-1.75(\mathrm{~m}, 2 \mathrm{H}), 1.31(\mathrm{~s}, 3 \mathrm{H}), 1.05(\mathrm{~d}, J$ $=7.01 \mathrm{~Hz}, 3 \mathrm{H}) \mathrm{ppm} ;{ }^{13} \mathrm{C} \mathrm{NMR}\left(125 \mathrm{MHz}, \mathrm{CDCl}_{3}\right): \delta 204.6,144.2,111.8,79.6,78.6,51.9,37.4$, 32.2, 26.4, 23.4 ppm; HRMS (ESI): $m / z$ calcd for $\mathrm{C}_{22} \mathrm{H}_{38} \mathrm{O}_{4} \mathrm{Si}[\mathrm{M}+\mathrm{Na}]^{+}$: 417.2437, found 417.2426.

(6R,7S,E)-7-((tert-Butyldimethylsilyl)oxy)-1-((2R,5R)-5-((tert-butyldiphenylsilyl)oxy) methyl)tetrahydrofuran-2-yl)-11-((4-methoxybenzyl)oxy)-6-methylundec-4-en-3-one (4):

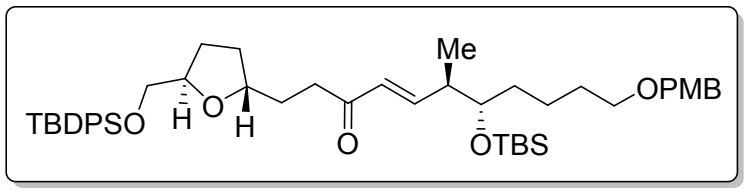

To a stirred solution of the keto phosphonate $5(0.157 \mathrm{~g}, 0.303 \mathrm{mmol})$ in $\mathrm{MeCN}(15 \mathrm{~mL})$, was added DIPEA (39 mg, $0.303 \mathrm{mmol})$ at $0{ }^{\circ} \mathrm{C}$ under nitrogen atmosphere. $\mathrm{LiCl}(13 \mathrm{mg}, 0.303$ mmol) followed by aldehyde $6(120 \mathrm{mg}, 0.303 \mathrm{mmol})$ were slowly added to the reaction mixture and stirred at the same temperature for $1 \mathrm{~h}$. The reaction was allowed to warm to room temperature and the stirring was then continued for $8 \mathrm{~h}$. After complete consumption of the starting material (monitored by TLC), the reaction mixture was quenched with saturated $\mathrm{NH}_{4} \mathrm{Cl}$ $(15 \mathrm{~mL})$, diluted with ethyl acetate $(30 \mathrm{~mL})$. The organic layer was separated and the aqueous layer was extracted with ethyl acetate $(2 \times 30 \mathrm{~mL})$. The combined organic layers were washed with brine $(50 \mathrm{~mL})$, dried over anhydrous $\mathrm{Na}_{2} \mathrm{SO}_{4}$ and was concentrated under reduced pressure 
to get the crude product which on purification by silica gel column chromatography (ethyl acetate/hexane $=1: 8)$ furnished the desired ketone $5(0.216 \mathrm{~g}, 91 \%)$ as a colorless liquid. $[\alpha]_{\mathrm{D}}{ }^{25}$ +4.6 (c 1.0, $\left.\mathrm{CHCl}_{3}\right)$; IR (KBr): $v_{\max } 2931,2857,1669,1249,1106,701 \mathrm{~cm}^{-1} ;{ }^{1} \mathrm{H}$ NMR $(400$ $\left.\mathrm{MHz}, \mathrm{CDCl}_{3}\right): \delta 7.71-7.65(\mathrm{~m}, 4 \mathrm{H}), 7.44-7.33(\mathrm{~m}, 6 \mathrm{H}), 7.25(\mathrm{~d}, J=8.55 \mathrm{~Hz}, 2 \mathrm{H}), 6.87(\mathrm{~d}, J=$ $8.682 \mathrm{H}), 6.82(\mathrm{dd}, J=16.01,7.82 \mathrm{~Hz}, 1 \mathrm{H}), 6.07(\mathrm{dd}, J=16.01,0.85 \mathrm{~Hz}, 1 \mathrm{H}), 4.41(\mathrm{~s}, 2 \mathrm{H}), 4.15$ $-4.08(\mathrm{~m}, 1 \mathrm{H}), 3.99-3.91(\mathrm{~m}, 1 \mathrm{H}), 3.79(\mathrm{~s}, 3 \mathrm{H}), 3.68-3.57(\mathrm{~m}, 3 \mathrm{H}), 3.45-3.37(\mathrm{~m}, 2 \mathrm{H}), 2.78$ $-2.68(\mathrm{~m}, 1 \mathrm{H}), 2.64-2.54(\mathrm{~m}, 1 \mathrm{H}), 2.49-2.40(\mathrm{~m}, 1 \mathrm{H}), 2.06-1.96(\mathrm{~m}, 2 \mathrm{H}), 1.88-1.70(\mathrm{~m}$, $3 \mathrm{H}), 1.60-1.48(\mathrm{~m}, 3 \mathrm{H}), 1.48-1.24(\mathrm{~m}, 4 \mathrm{H}), 1.05(\mathrm{~s}, 9 \mathrm{H}), 1.04(\mathrm{~d}, J=0.9 \mathrm{~Hz}, 3 \mathrm{H}), 0.88(\mathrm{~s}$, 9H), 0.03 (s, 6H) ppm; ${ }^{13} \mathrm{C}$ NMR (125 MHz, $\left.\mathrm{CDCl}_{3}\right): \delta 200.3,159.1,149.1,135.6,133.7,130.7$, $130.2,129.5,129.2,127.6,113.7,78.9,78.7,75.3,72.5,69.9,66.5,52.2,41.9,36.7,34.1,31.8$ 29.9, 29.9, 28.1, 26.8, 25.9, 22.1, 19.2, 18.1, 15.4, -4.3, -4.5 ppm; HRMS (ESI): $m / z$ calcd. for $\mathrm{C}_{47} \mathrm{H}_{70} \mathrm{O}_{6} \mathrm{Si}_{2}\left[\mathrm{M}+\mathrm{NH}_{4}\right]^{+}:$804.5049, found 804.5066.

(4S,5R,6R,7S)-7-((tert-Butyldimethylsilyl)oxy)-1-((2R,5R)-5-(((tert-butyldiphenylsilyl)oxy) methyl)tetrahydrofuran-2-yl)-4,5-dihydroxy-11-((4-methoxybenzyl)oxy)-6-methylundecan3-one (4):

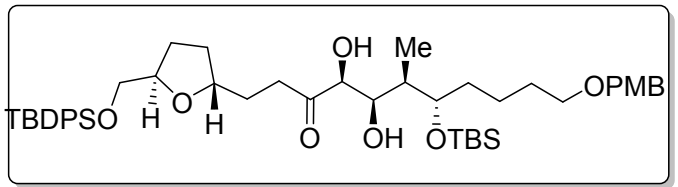

A mixture of $\mathrm{K}_{2} \mathrm{OSO}_{2}(\mathrm{OH})_{2}\left(0.33 \mathrm{mg}, 0.0009 \mathrm{mmol}, 0.006\right.$ equiv), (DHQD) ${ }_{2} \mathrm{PHAL}$ (1.78 mg, 0.002 mmol, 0.015 equiv), $\mathrm{K}_{3} \mathrm{Fe}(\mathrm{CN})_{6}\left(150 \mathrm{mg}, 0.45 \mathrm{mmol}, 3.0\right.$ equiv.), $\mathrm{K}_{2} \mathrm{CO}_{3}$ (63 mg, 0.45 mmol, 3.0 equiv) and $\mathrm{MeSO}_{2} \mathrm{NH}_{2}\left(14.5 \mathrm{mg}, 0.152 \mathrm{mmol}, 1.0\right.$ equiv) in $1: 1 t-\mathrm{BuOH} / \mathrm{H}_{2} \mathrm{O}(50 \mathrm{~mL})$ was stirred for $30 \mathrm{~min}$ at room temperature. The mixture was slowly poured into a $4{ }^{\circ} \mathrm{C}$ solution of 5 (120 mg, $0.152 \mathrm{mmol}, 1.0$ equiv) in $t-\mathrm{BuOH} / \mathrm{H}_{2} \mathrm{O}(30 \mathrm{~mL})$. Then, the reaction was stirred at $4{ }^{\circ} \mathrm{C}$ for $36 \mathrm{~h}$. After completion of reaction (TLC), solid $\mathrm{Na}_{2} \mathrm{~S}_{2} \mathrm{O}_{3}(0.5 \mathrm{~g})$ was added and the 
mixture stirred for $1.5 \mathrm{~h}$ while warming to room temperature (color changed from orange to dark green). After phase separation, the aqueous layer was extracted with ethyl acetate $(3 \times 20 \mathrm{~mL})$. The combined organic layers were dried over $\mathrm{Na}_{2} \mathrm{SO}_{4}$, concentrated under reduced presure to provide crude product which was purified by silica gel column chromatography (ethyl acetate/hexane $=3: 7)$ to furnish $20(100 \mathrm{mg}, 80 \%) \cdot[\alpha]_{\mathrm{D}}{ }^{25}+15.25\left(c 0.8, \mathrm{CHCl}_{3}\right) ; \mathrm{IR}(\mathrm{KBr}): v_{\max }$ $3468,2931,2857,1714,1471,1112,704 \mathrm{~cm}^{-1} ;{ }^{1} \mathrm{H}$ NMR $\left(400 \mathrm{MHz}, \mathrm{CDCl}_{3}\right): \delta 7.70-7.64(\mathrm{~m}$, 4H), $7.44-7.34(\mathrm{~m}, 6 \mathrm{H}), 7.25(\mathrm{~d}, J=8.7 \mathrm{~Hz}, \mathrm{H}), 6.87(\mathrm{~d}, J=8.7 \mathrm{~Hz}, 2 \mathrm{H}), 4.42(\mathrm{~s}, 2 \mathrm{H}), 4.20-$ $4.04(\mathrm{~m}, 3 \mathrm{H}), 3.99-3.90(\mathrm{~m}, 1 \mathrm{H}), 3.88-3.81(\mathrm{~m}, 1 \mathrm{H}), 3.79(\mathrm{~s}, 3 \mathrm{H}), 3.77-3.71(\mathrm{~m}, 1 \mathrm{H}), 3.69-$ $3.56(\mathrm{~m}, 3 \mathrm{H}), 3.45-3.69(\mathrm{~m}, 2 \mathrm{H}), 3.1-3.0(\mathrm{~m}, 1 \mathrm{H}), 2.74-2.64(\mathrm{~m}, 1 \mathrm{H}), 2.08-1.94(\mathrm{~m}, 2 \mathrm{H})$, $1.93-1.72(\mathrm{~m}, 4 \mathrm{H}), 1.68-1.44(\mathrm{~m}, 7 \mathrm{H}), 1.43-1.30(\mathrm{~m}, 1 \mathrm{H}), 1.05(\mathrm{~s}, 9 \mathrm{H}), 1.03(\mathrm{~d}, J=7.2 \mathrm{~Hz}$ $3 \mathrm{H}), 0.88(\mathrm{~s}, 9 \mathrm{H}), 0.08-0.06(\mathrm{~m}, 6 \mathrm{H}) \mathrm{ppm} ;{ }^{13} \mathrm{C} \mathrm{NMR}\left(125 \mathrm{MHz}, \mathrm{CDCl}_{3}\right): \delta$ 11.6, 159.0, 135.6, $133.6,130.7,130.6,129.6,129.2,127.6,113.7,78.9,78.6,76.3,72.6,71.5,70.1,69.9,66.4$, $55.2,40.3,39.5,35.3,33.8,31.7,29.8,29.5,28.0,26.8,25.9,22.0,21.6,19.2,18.0,12.0,-4.4$ -4.4, -4.6, -4.7 ppm; HRMS (ESI): $m / z$ calcd. for $\mathrm{C}_{47} \mathrm{H}_{72} \mathrm{O}_{8} \mathrm{Si}_{2}[\mathrm{M}+\mathrm{Na}]^{+}: 843.4658$, found 843.4656.

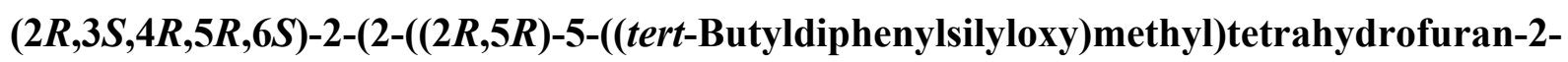
yl)ethyl)-2-methoxy-6-(3-(4-methoxybenzyloxy)propyl)-5-methyltetrahydro-2H-pyran-3,4diol (3):

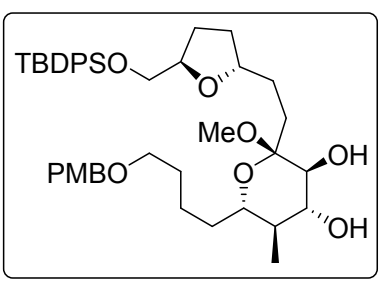

To the solution of keto diol $20(60 \mathrm{mg}, 0.073 \mathrm{mmol})$ in $\mathrm{MeOH}(20 \mathrm{~mL})$, pyridinium $p$ toluenesulfonate $(1.8 \mathrm{mg}, 0.0073 \mathrm{mmol})$ was added at $0{ }^{\circ} \mathrm{C}$. The resulting solution was stirred for 
$12 \mathrm{~h}$ at room temperature. After completion of the reaction (TLC), triethylamine ( $0.5 \mathrm{~mL})$ was added and concentrated under reduced pressure to give crude residue which was purified by silica gel chromatography (ethyl acetate/hexane $=3: 7$ ) to furnish $\mathrm{C} 1-\mathrm{C} 16$ fragment of formosalide $3(42 \mathrm{mg}, 82 \%)$ as a colorless liquid. $[\alpha]_{\mathrm{D}}{ }^{25}-31.2\left(c 1.0, \mathrm{CHCl}_{3}\right)$; IR $(\mathrm{KBr}): v_{\max }$ 3424, 2933, 2859, 1513, 1428, 1112, $705 \mathrm{~cm}^{-1} ;{ }^{1} \mathrm{H}$ NMR (500 MHz, $\left.\mathrm{CDCl}_{3}\right): \delta 7.71-7.65(\mathrm{~m}$, 4H), $7.44-7.34(\mathrm{~m}, 6 \mathrm{H}), 7.25(\mathrm{~d}, J=7.5 \mathrm{~Hz}, 2 \mathrm{H}), 6.87(\mathrm{~d}, J=8.7 \mathrm{~Hz}, 2 \mathrm{H}), 4.43(\mathrm{~s}, 2 \mathrm{H}), 4.19-$ $4.09(\mathrm{~m}, 1 \mathrm{H}), 3.95-3.88(\mathrm{~m}, 1 \mathrm{H}), 3.79(\mathrm{~s}, 3 \mathrm{H}), 3.64(\mathrm{dd}, J=4.8,0.9 \mathrm{~Hz}, 2 \mathrm{H}), 3.46-3.27(\mathrm{~m}$, 4H), $3.18(\mathrm{~s}, 3 \mathrm{H}), 2.58(\mathrm{~d}, J=8.8 \mathrm{~Hz}, 1 \mathrm{H}), 2.40(\mathrm{br} \mathrm{s}, 1 \mathrm{H}), 2.05-1.94(\mathrm{~m}, 2 \mathrm{H}), 1.87-1.73(\mathrm{~m}$, 4H), $1.64-1.33(\mathrm{~m}, 8 \mathrm{H}), 1.05(\mathrm{~s}, 9 \mathrm{H}), 0.95(\mathrm{~d}, J=6.6 \mathrm{~Hz}, 3 \mathrm{H}) \mathrm{ppm} ;{ }^{13} \mathrm{C}$ NMR $(125 \mathrm{MHz}$, $\left.\mathrm{CDCl}_{3}\right): \delta 159.1,135.6,133.7,129.5,129.2,127.6,113.7,100.5,79.8,79.1,75.8,74.8,73.4$ $72.5,70.0,66.5,55.2,47.4,41.2,32.1,31.9,29.9,29.9,29.7,29.4,28.0,26.8,21.9,19.2,12.9$ ppm; HRMS (ESI): $m / z$ calcd. for $\mathrm{C}_{42} \mathrm{H}_{60} \mathrm{O}_{8} \mathrm{Si}[\mathrm{M}+\mathrm{Na}]^{+}:$743.3955, found 743.3945 . 

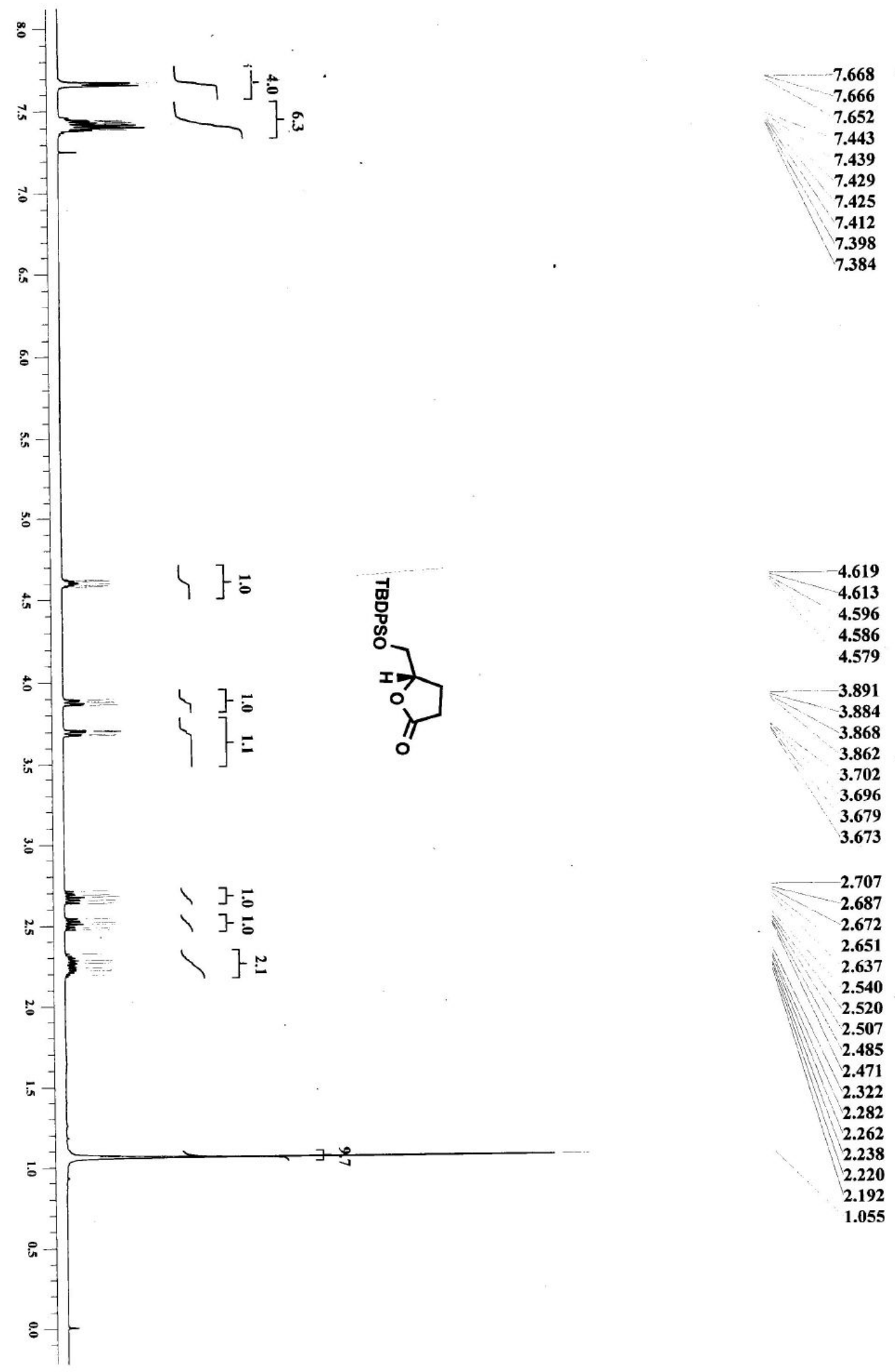

3.868
3.862

3.702

3.696

3.679

3.673

2.707

2.687

2.672

2.651

2.637

2.540

2.520

2.507

2.485

2.471

2.322

2.282

2.262

2.238

2.220

2.192

1.055

${ }^{1} \mathrm{H}$ NMR spectrum of $7\left(500 \mathrm{MHz}, \mathrm{CDCl}_{3}\right)$ 


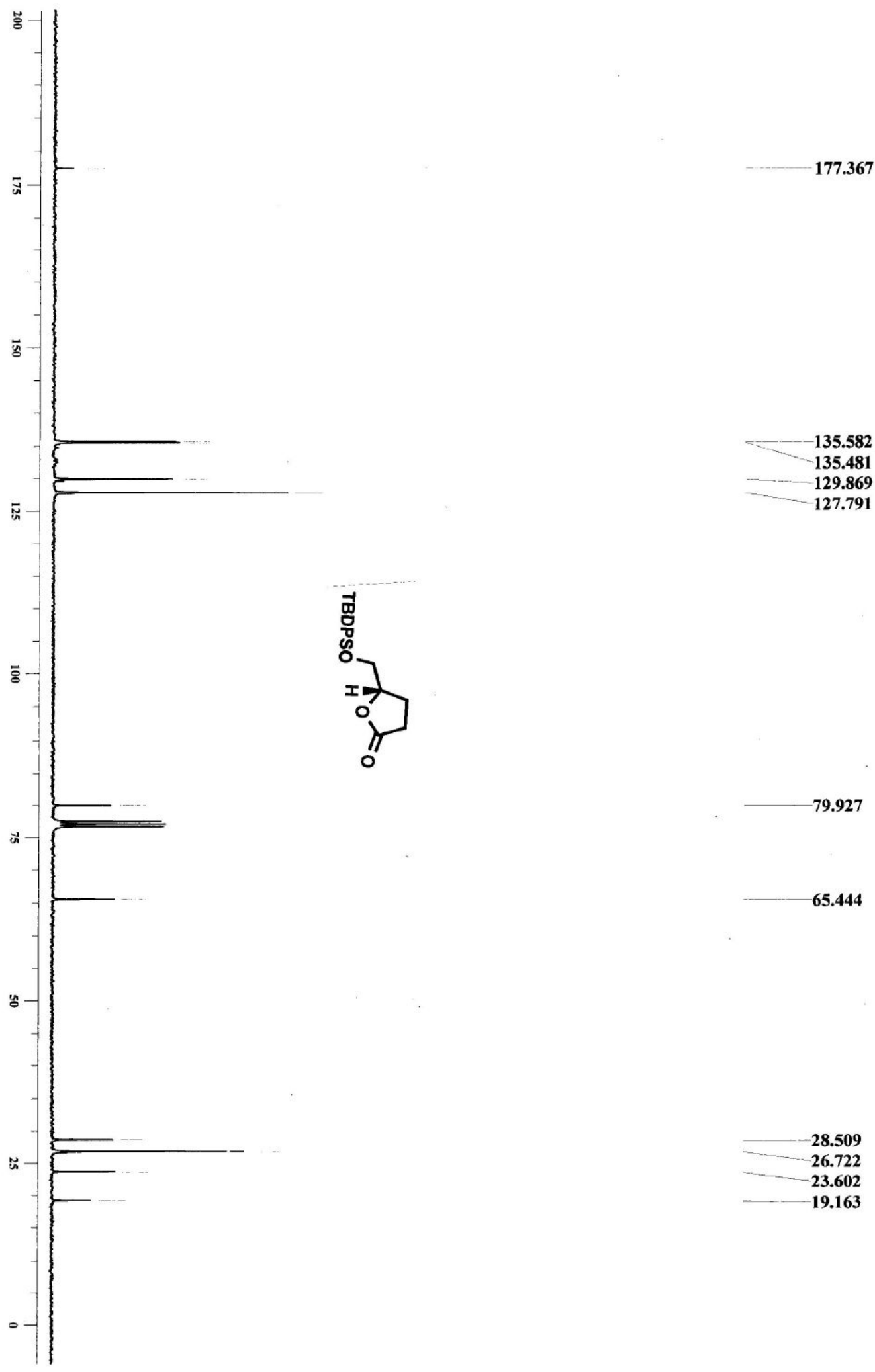

${ }^{13} \mathrm{C}$ NMR spectrum of $7\left(75 \mathrm{MHz}, \mathrm{CDCl}_{3}\right)$ 


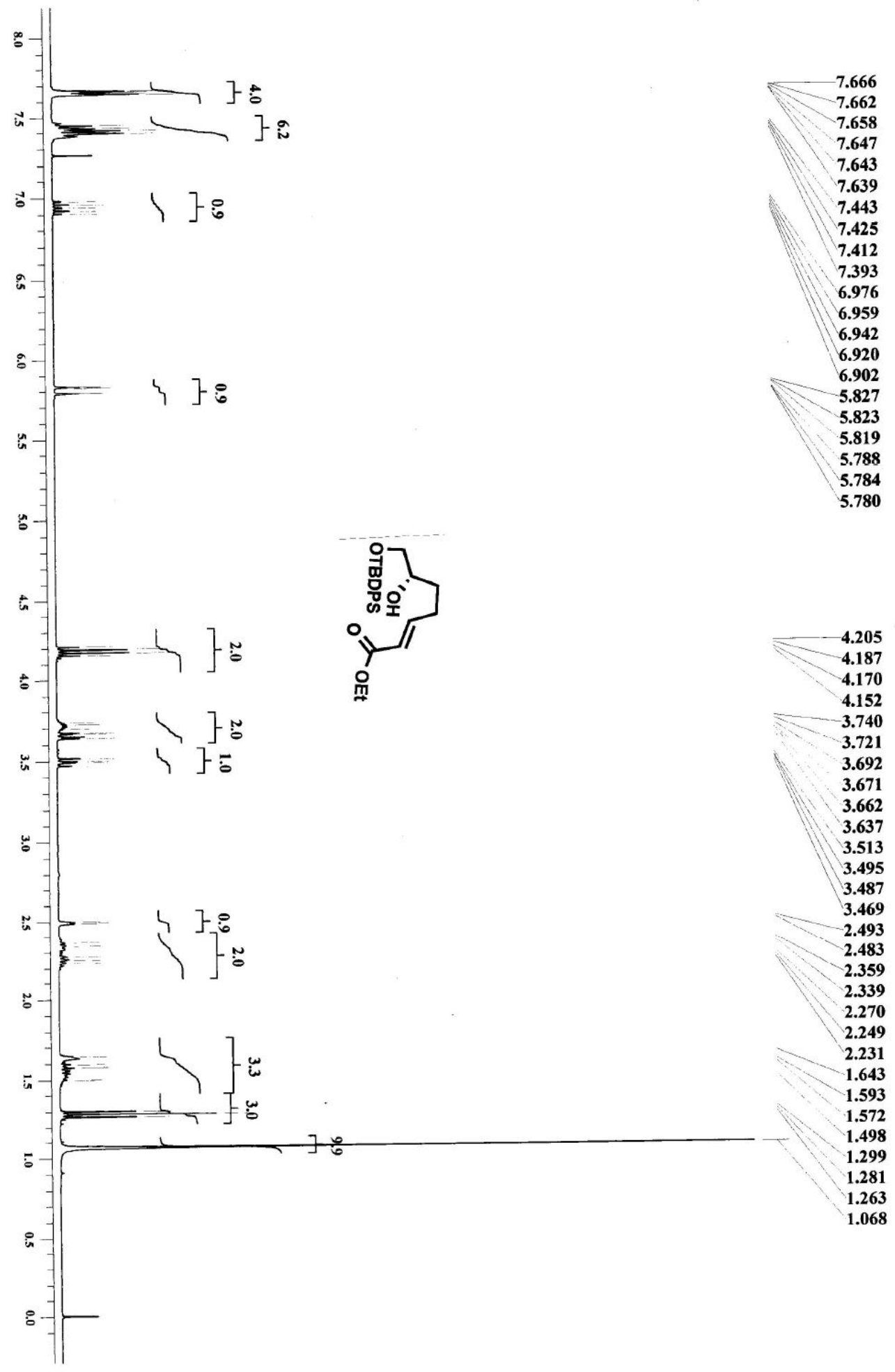

${ }^{1} \mathrm{H}$ NMR spectrum of $9\left(400 \mathrm{MHz}, \mathrm{CDCl}_{3}\right)$ 


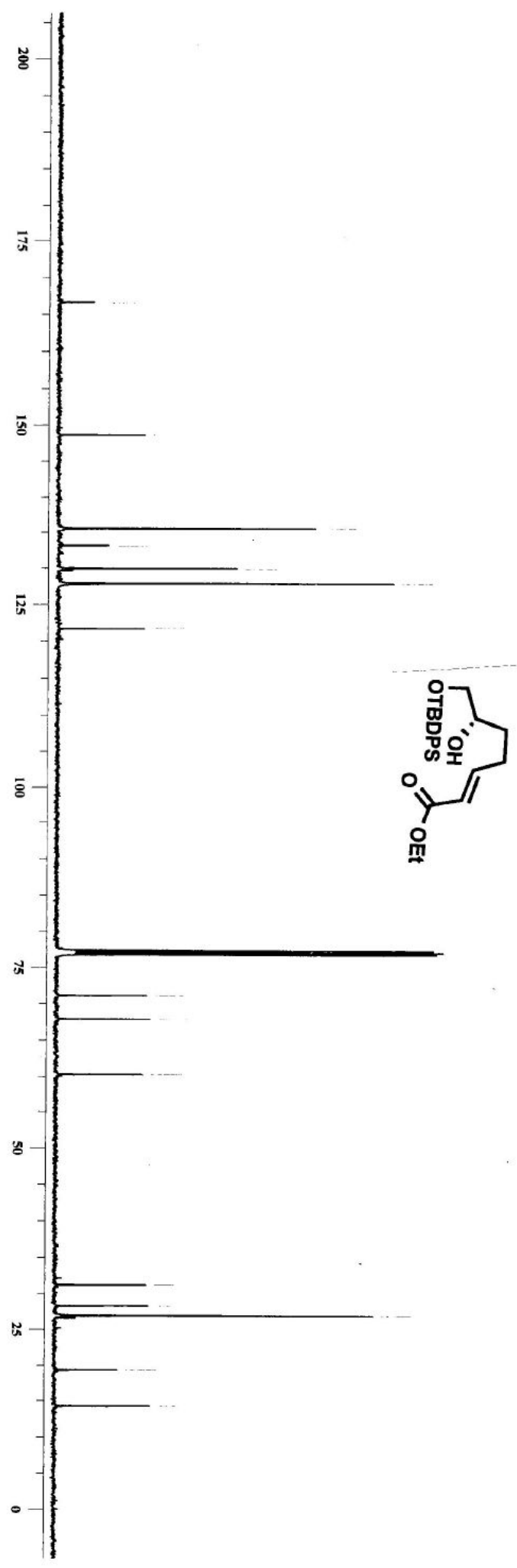

$-166.603$

$-148.477$
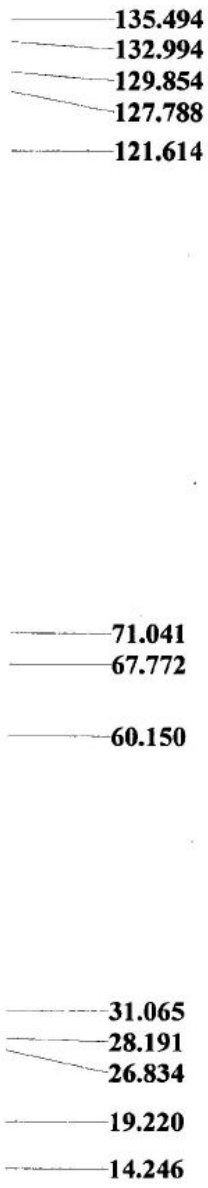

${ }^{13} \mathrm{C}$ NMR spectrum of $9\left(125 \mathrm{MHz}, \mathrm{CDCl}_{3}\right)$ 


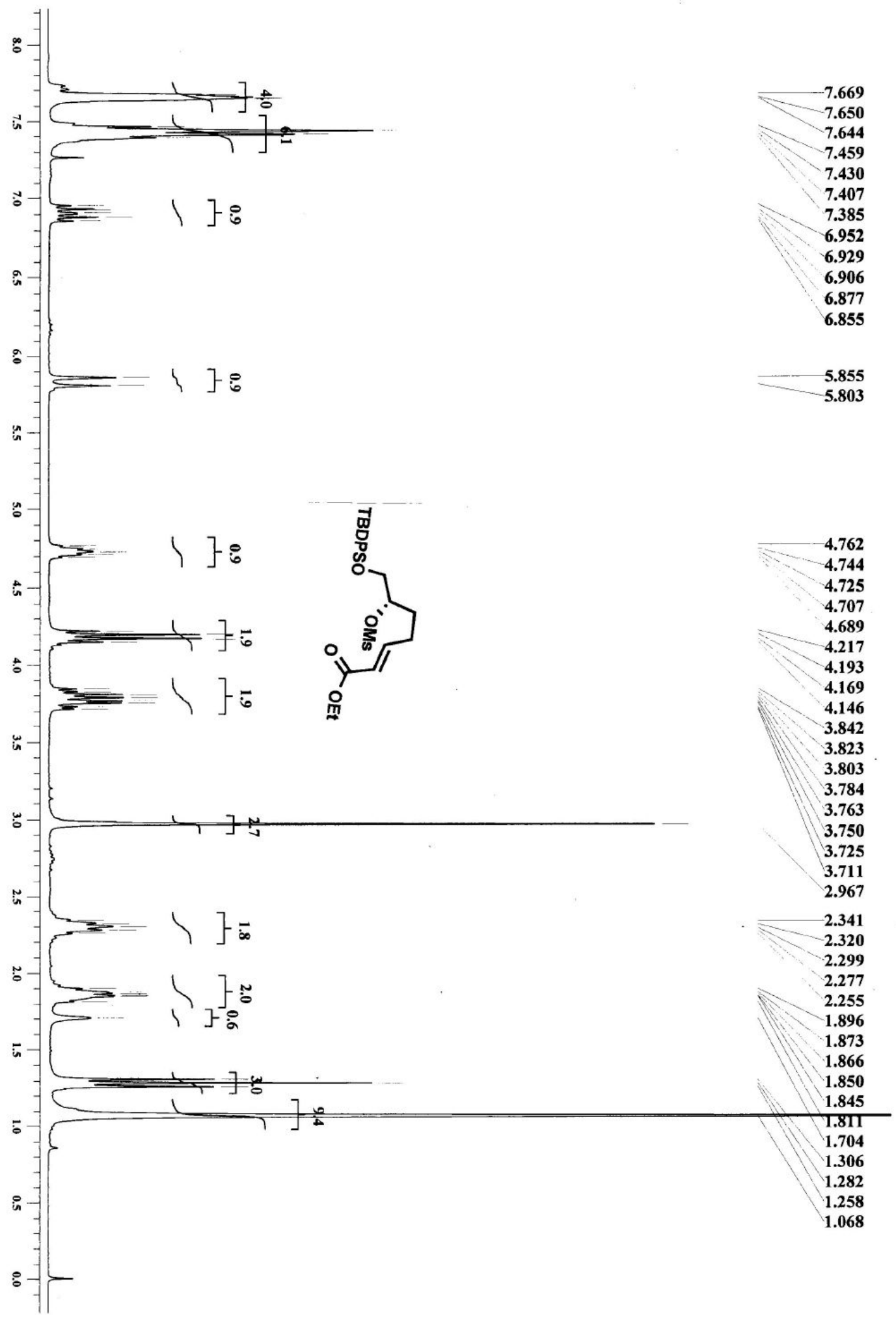

${ }^{1} \mathrm{H}$ NMR spectrum of $10\left(400 \mathrm{MHz}, \mathrm{CDCl}_{3}\right)$ 


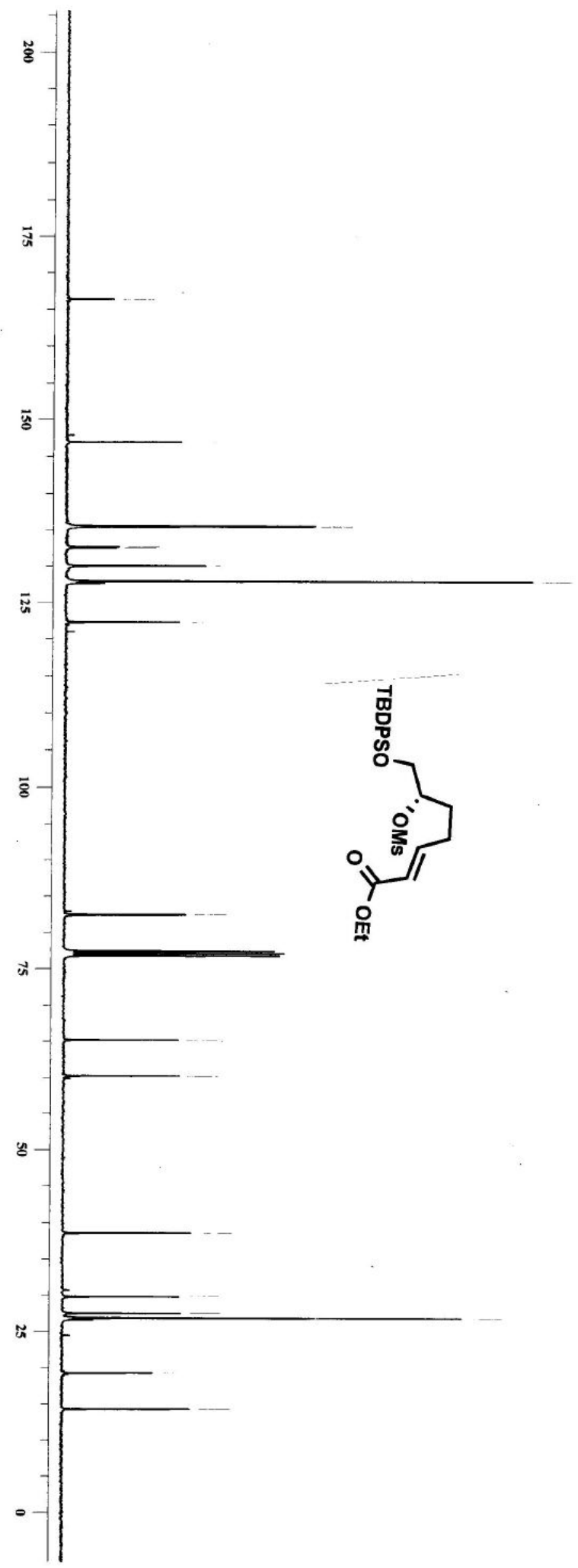

$-166.321$

146.884

$-135.503$

135.408

132.617

132.492

130.008

129.970

127.857

122.220

82.313

65.073

60.225

38.551

$\mathbf{2 9 . 7 2 7}$
-27.438

$-27.438$

26.770

$-19.149$

$-14.194$

${ }^{13} \mathrm{C}$ NMR spectrum of $\mathbf{1 0}\left(100 \mathrm{MHz}, \mathrm{CDCl}_{3}\right)$ 


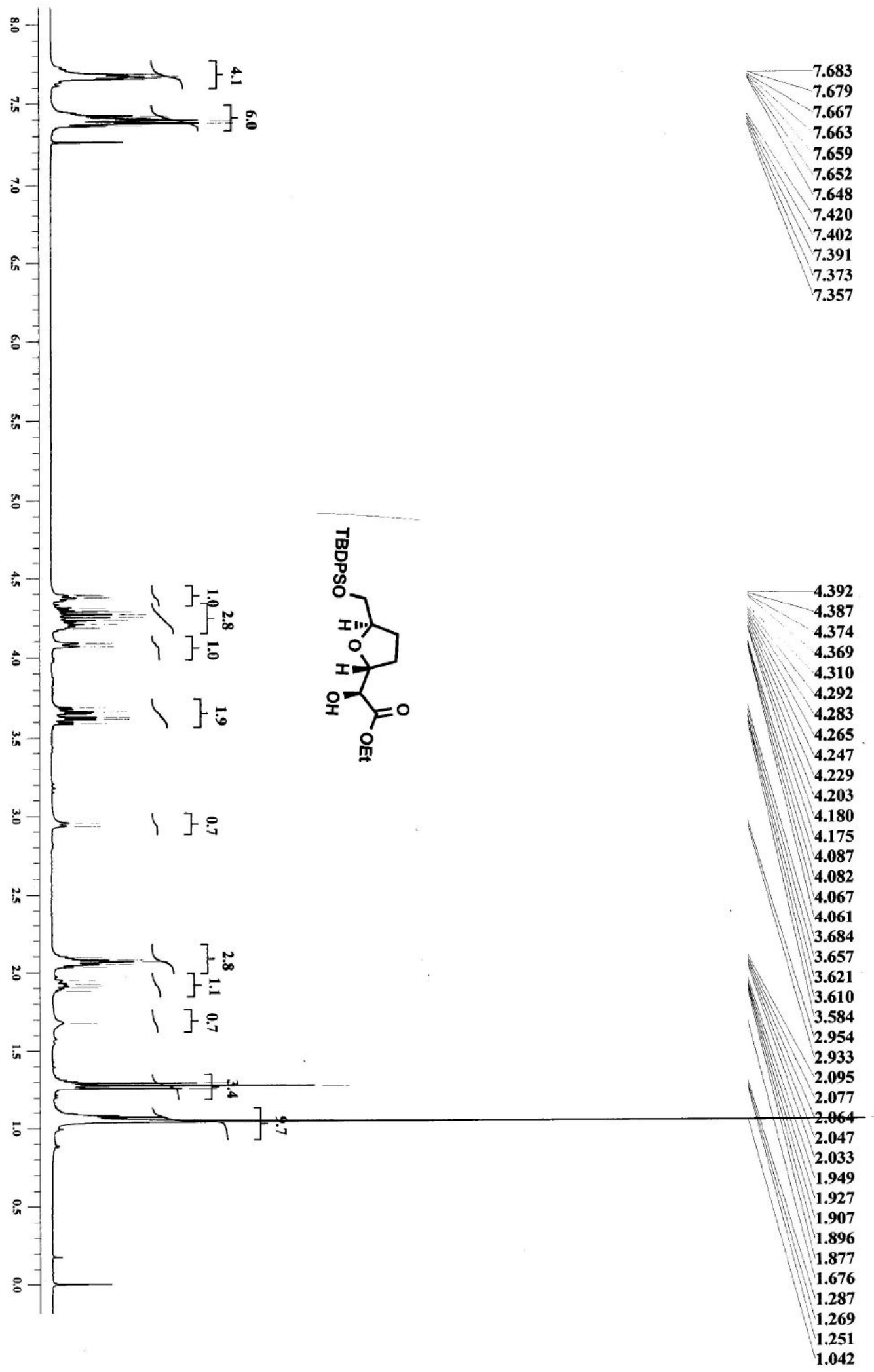

${ }^{1} \mathrm{H}$ NMR spectrum of $11\left(400 \mathrm{MHz}, \mathrm{CDCl}_{3}\right)$ 


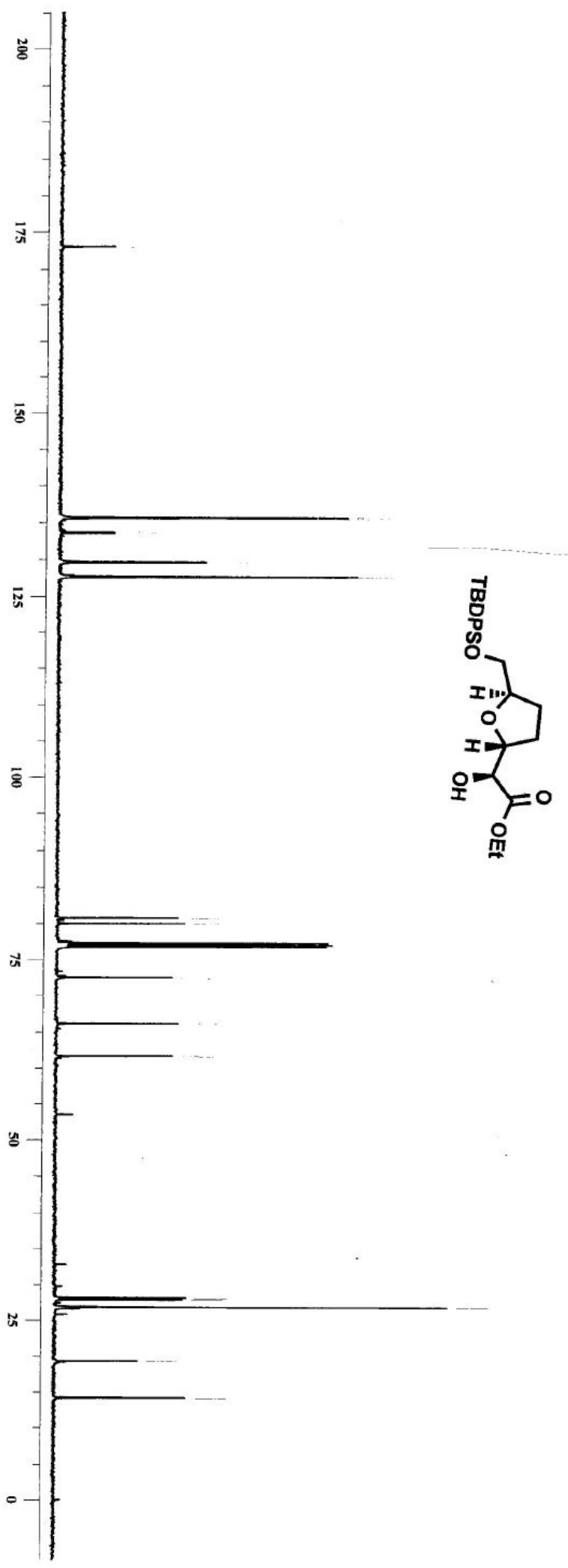

$-173.002$

135.571

135.535

133.556

133.521

129.573

129.543

127.601

127.570

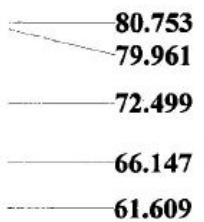

${ }^{13} \mathrm{C}$ NMR spectrum of $11\left(125 \mathrm{MHz}, \mathrm{CDCl}_{3}\right)$ 


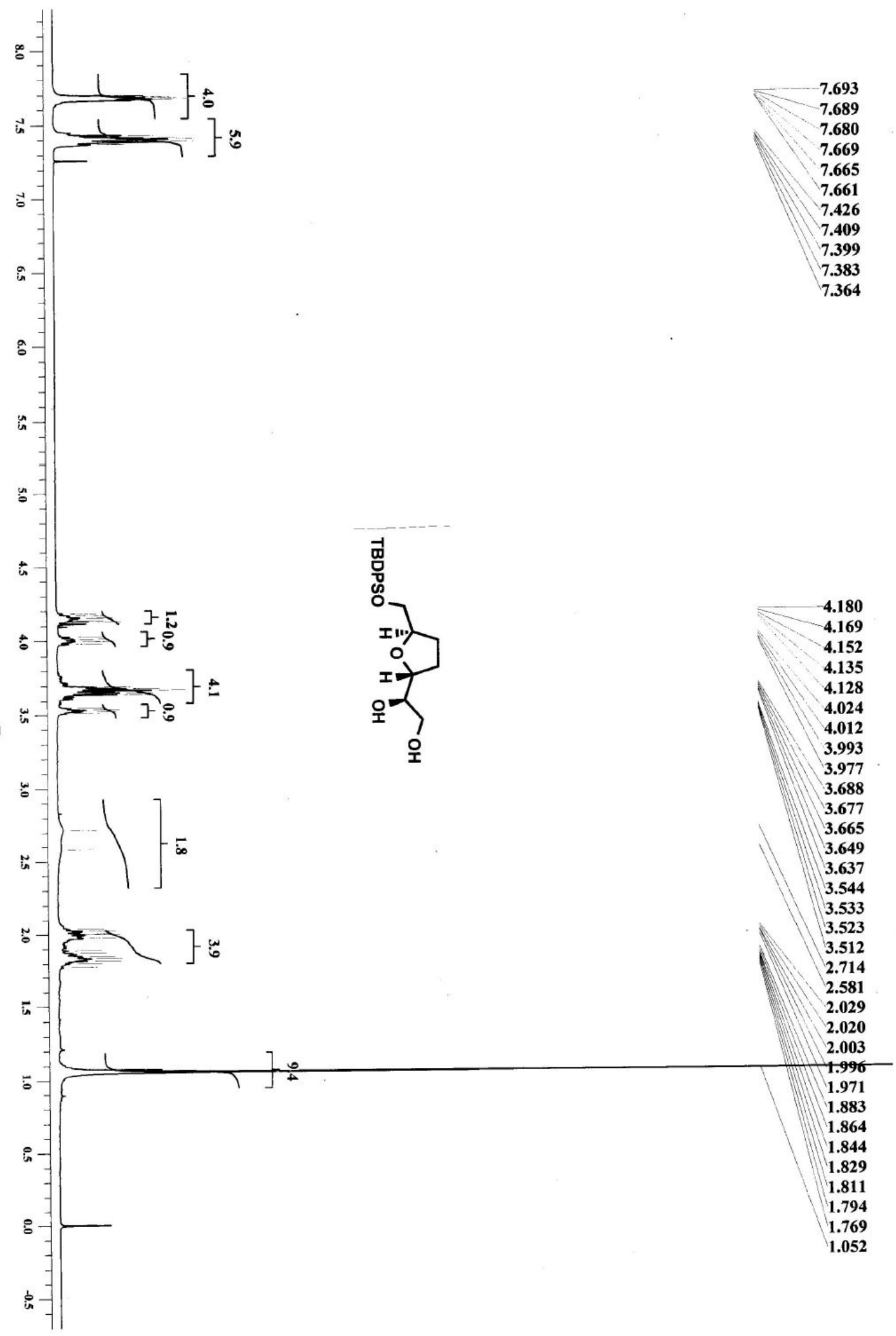

${ }^{1} \mathrm{H}$ NMR spectrum of $12\left(400 \mathrm{MHz}, \mathrm{CDCl}_{3}\right)$ 

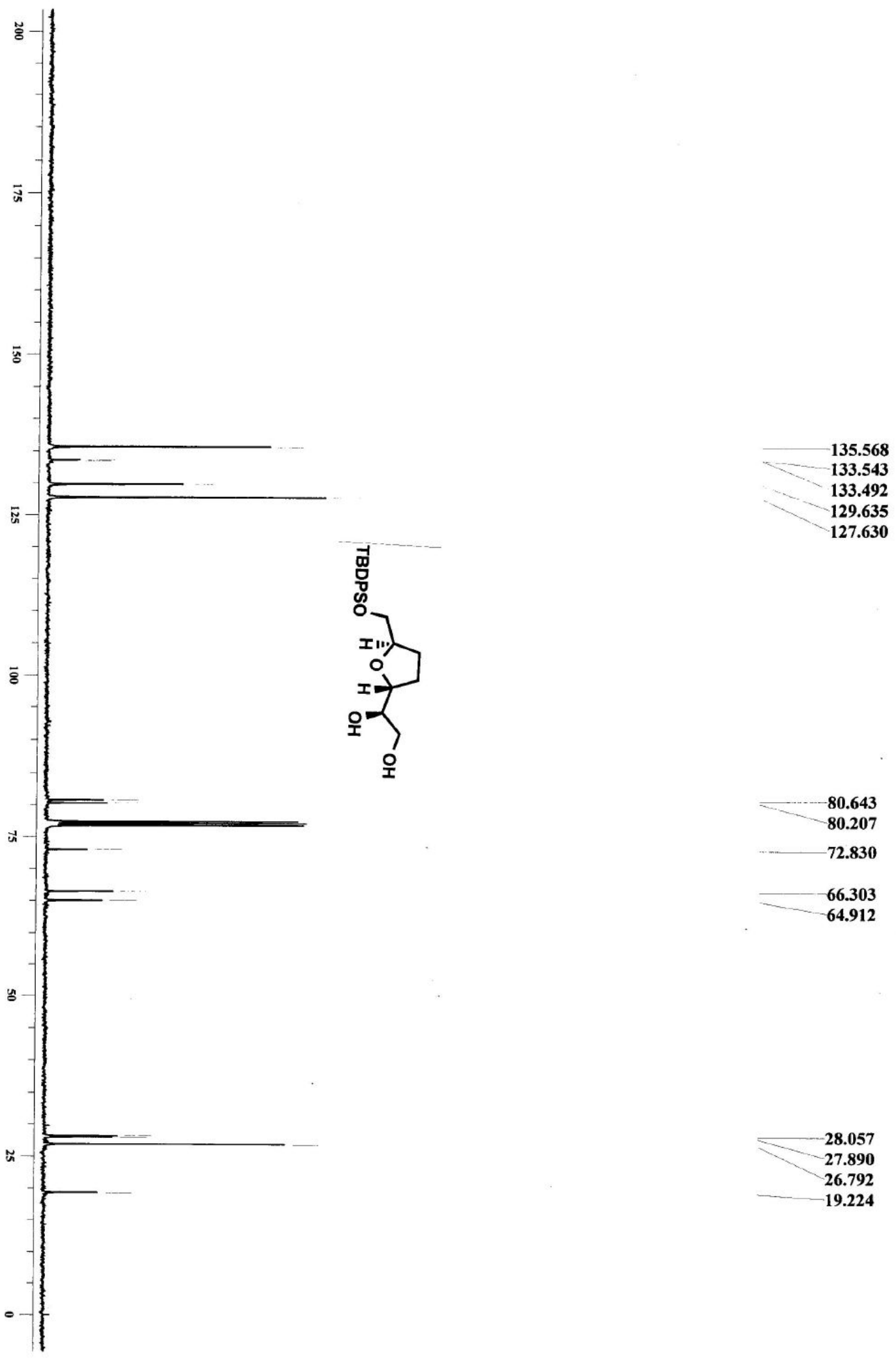

${ }^{13} \mathrm{C}$ NMR spectrum of $12\left(100 \mathrm{MHz}, \mathrm{CDCl}_{3}\right)$ 


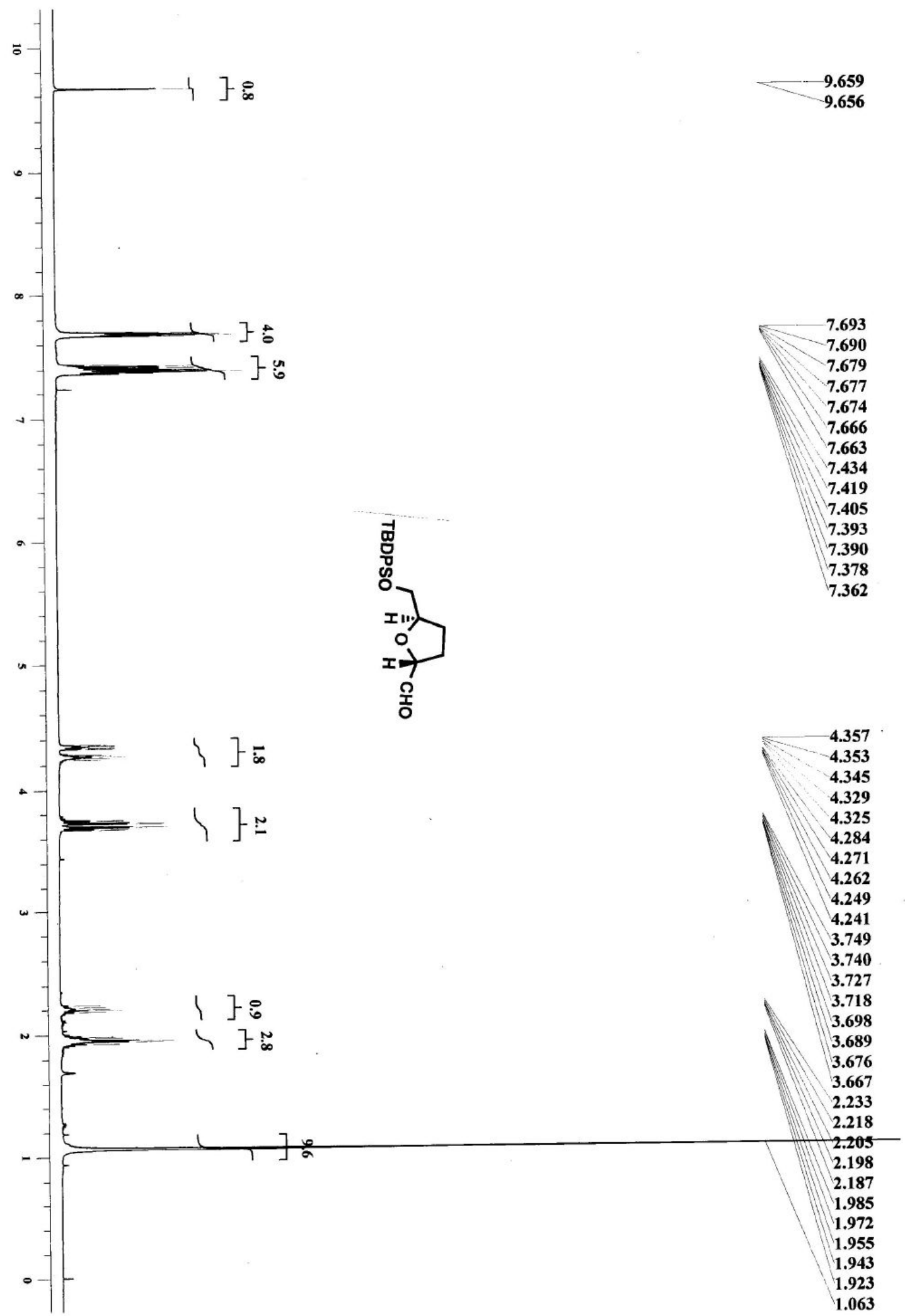

${ }^{1} \mathrm{H}$ NMR spectrum of $\mathbf{1 3}\left(500 \mathrm{MHz}, \mathrm{CDCl}_{3}\right)$ 


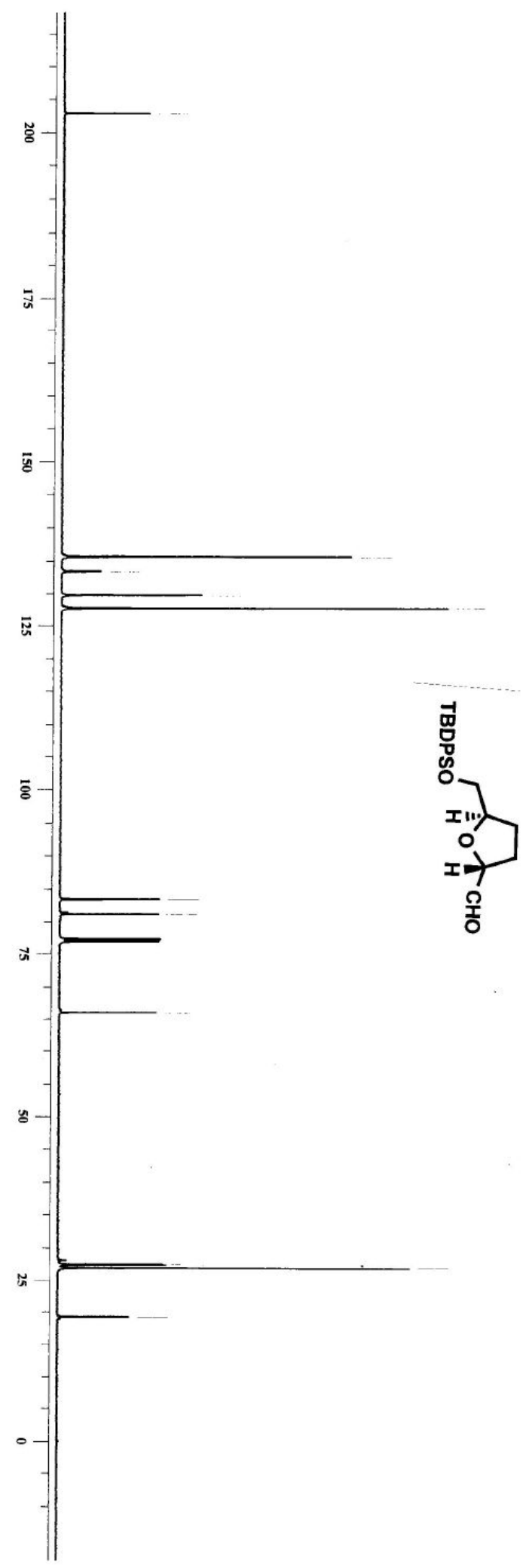

$-202.774$
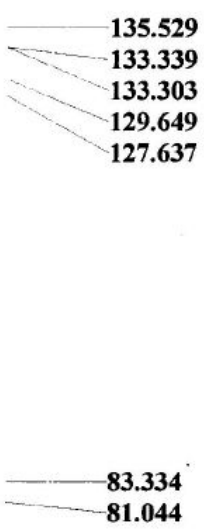

65.893

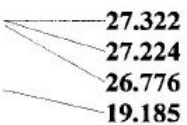

${ }^{13} \mathrm{C}$ NMR spectrum of $\mathbf{1 3}\left(125 \mathrm{MHz}, \mathrm{CDCl}_{3}\right)$ 


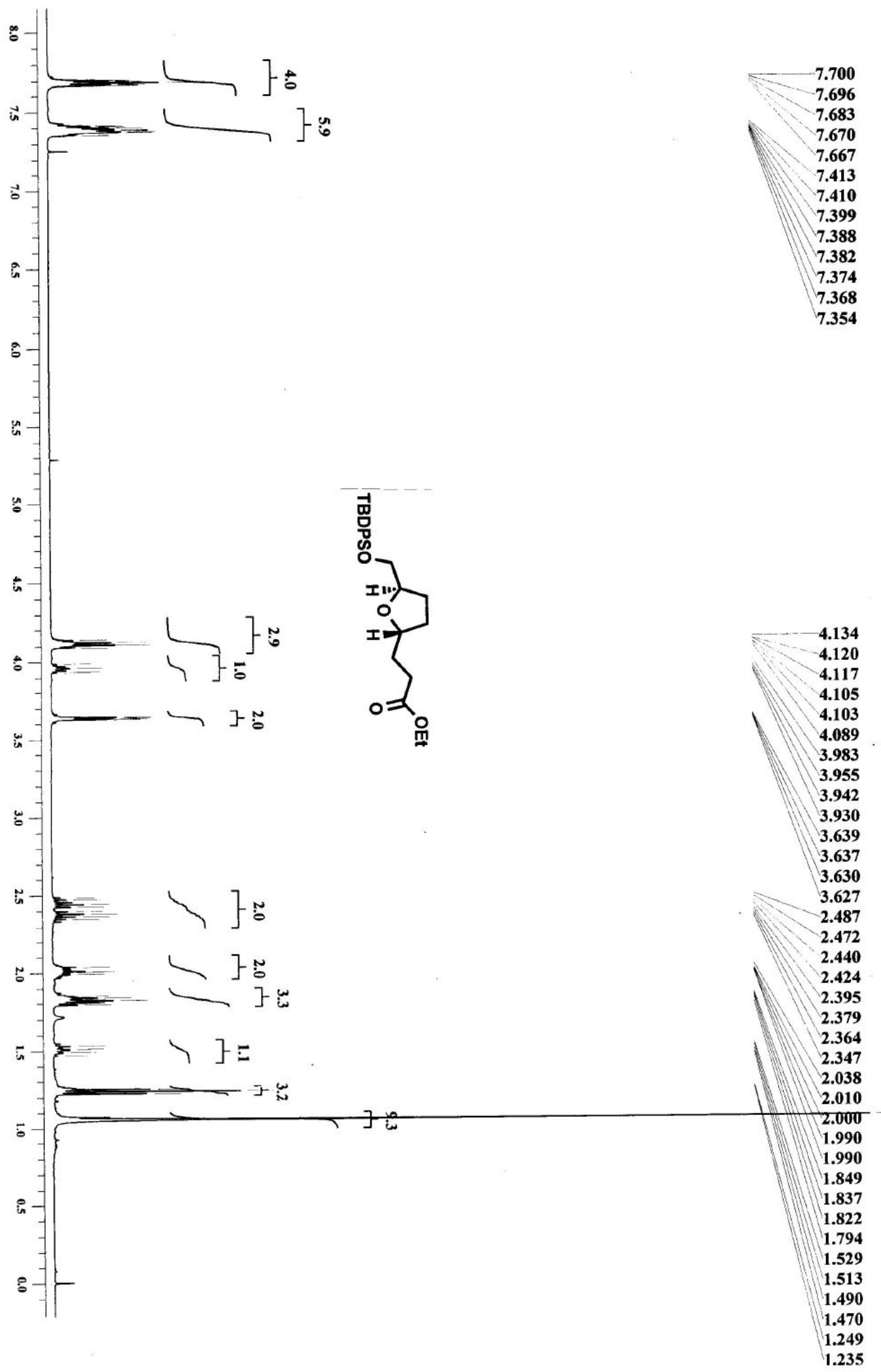

${ }^{1} \mathrm{H}$ NMR spectrum of $15\left(500 \mathrm{MHz}, \mathrm{CDCl}_{3}\right)$ 


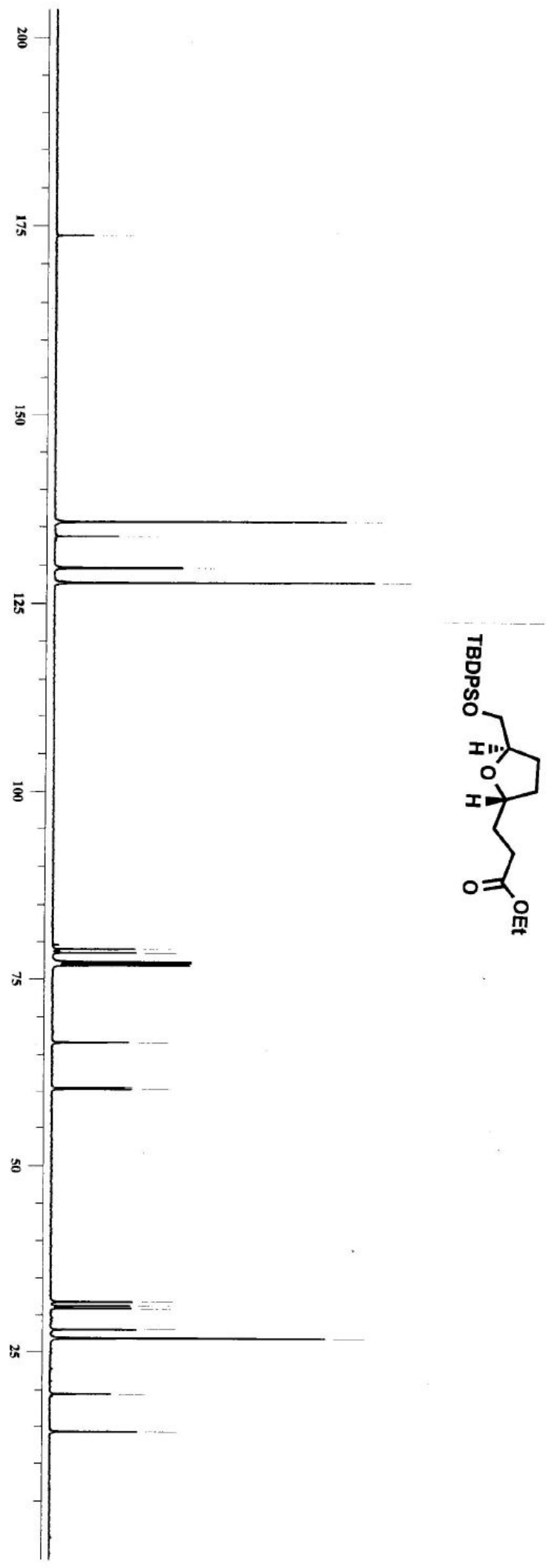

$-173.572$
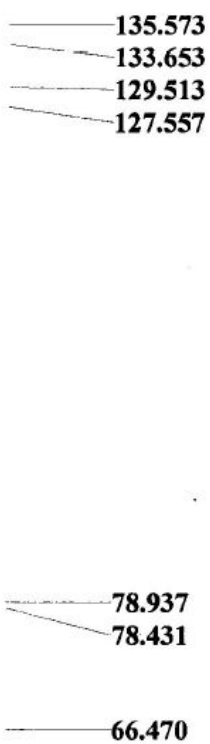

$-60.217$

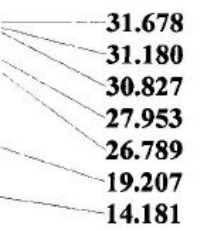

${ }^{13} \mathrm{C}$ NMR spectrum of $15\left(100 \mathrm{MHz}, \mathrm{CDCl}_{3}\right)$ 


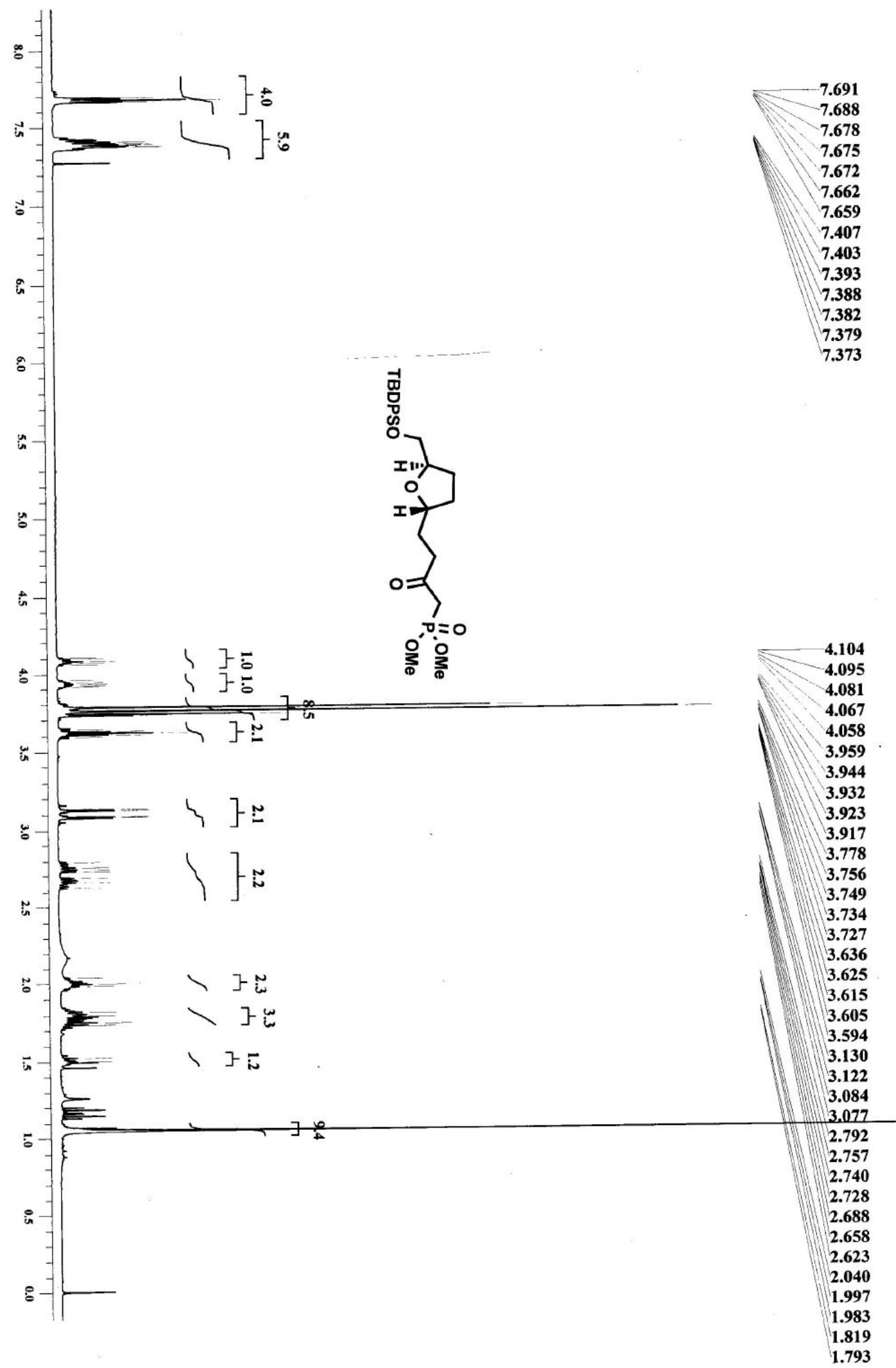

${ }^{1} \mathrm{H}$ NMR spectrum of $5\left(500 \mathrm{MHz}, \mathrm{CDCl}_{3}\right)$ 


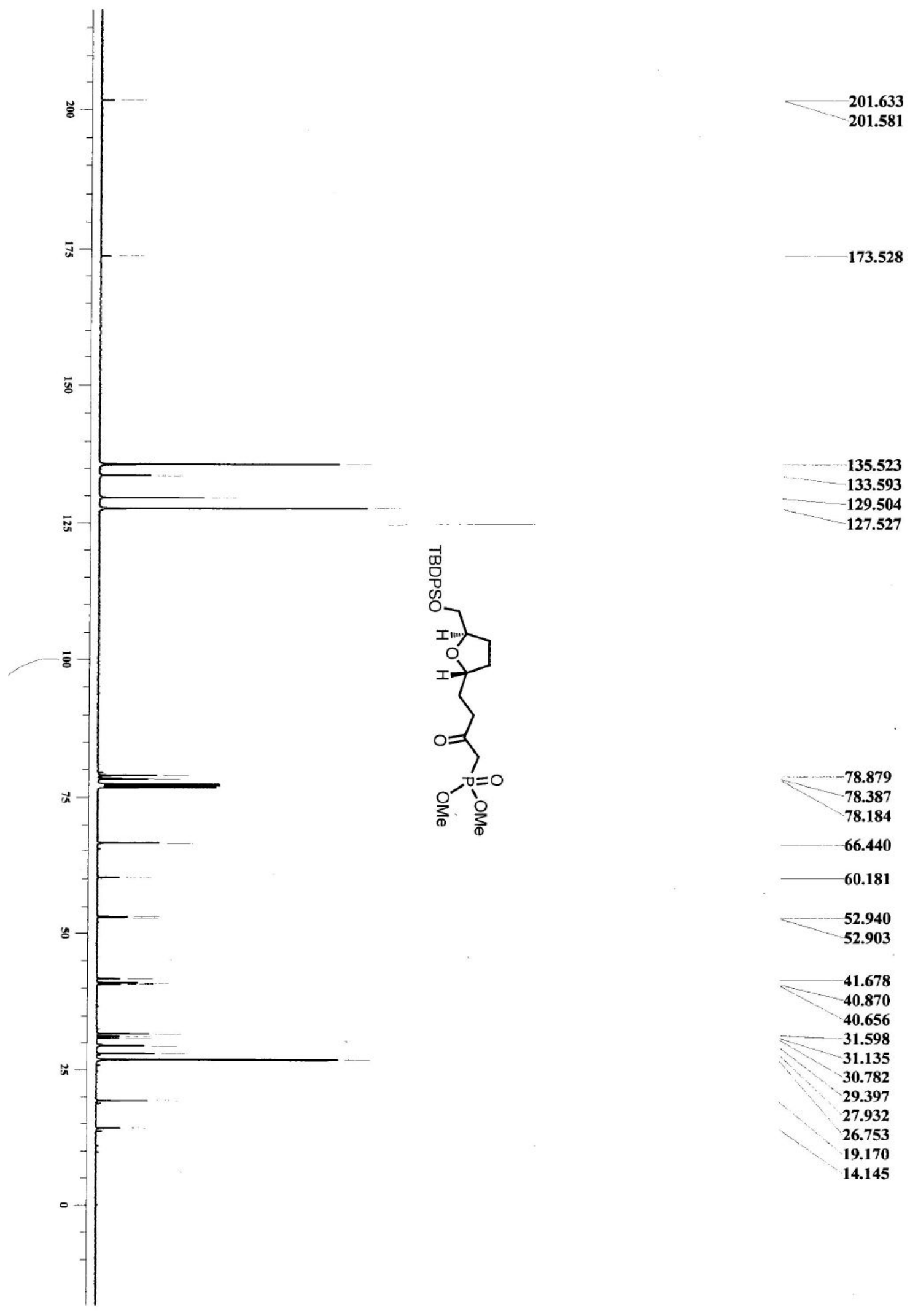

${ }^{13} \mathrm{C}$ NMR spectrum of $5\left(100 \mathrm{MHz}, \mathrm{CDCl}_{3}\right)$ 


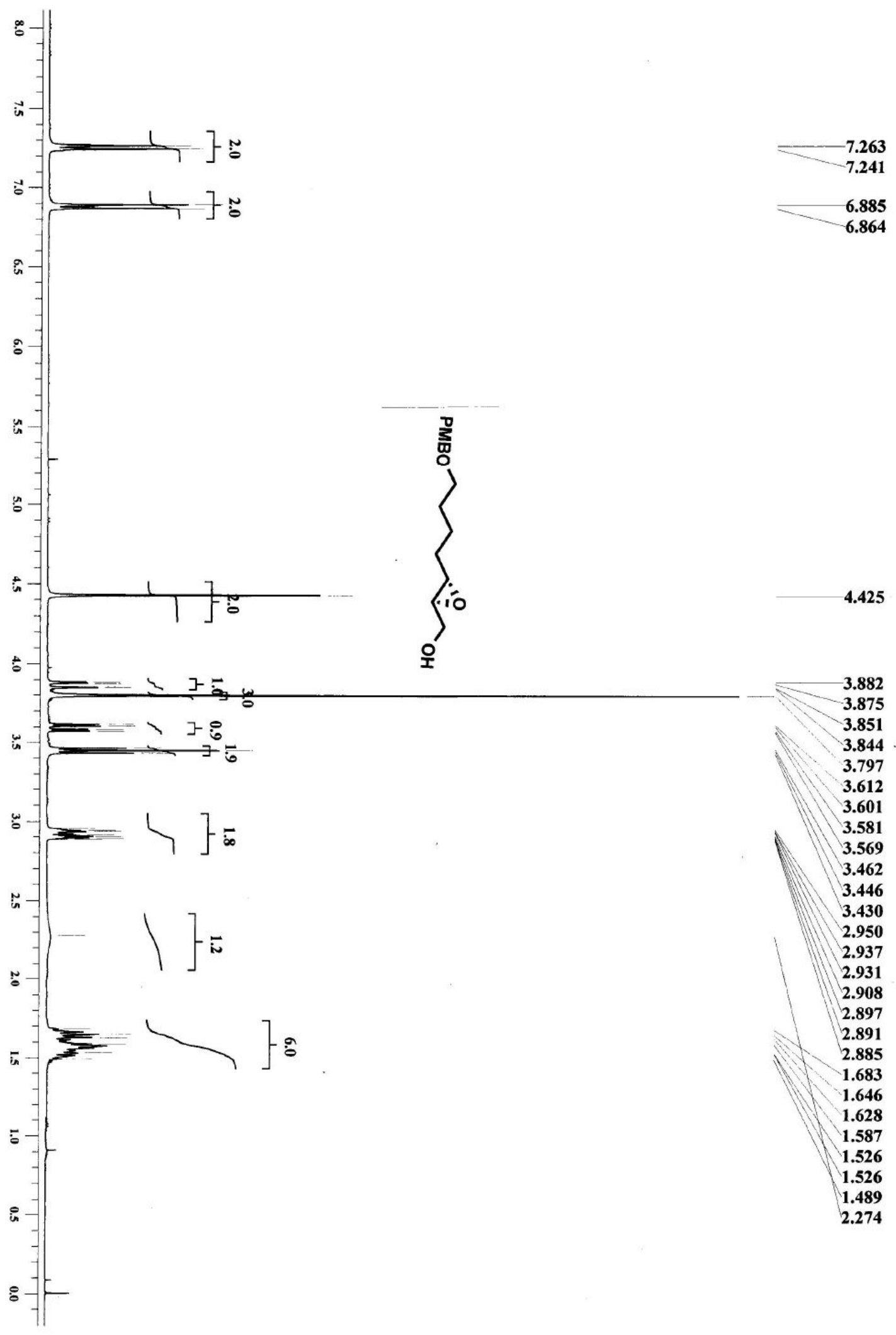

${ }^{1} \mathrm{H}$ NMR spectrum of $16\left(500 \mathrm{MHz}, \mathrm{CDCl}_{3}\right)$ 


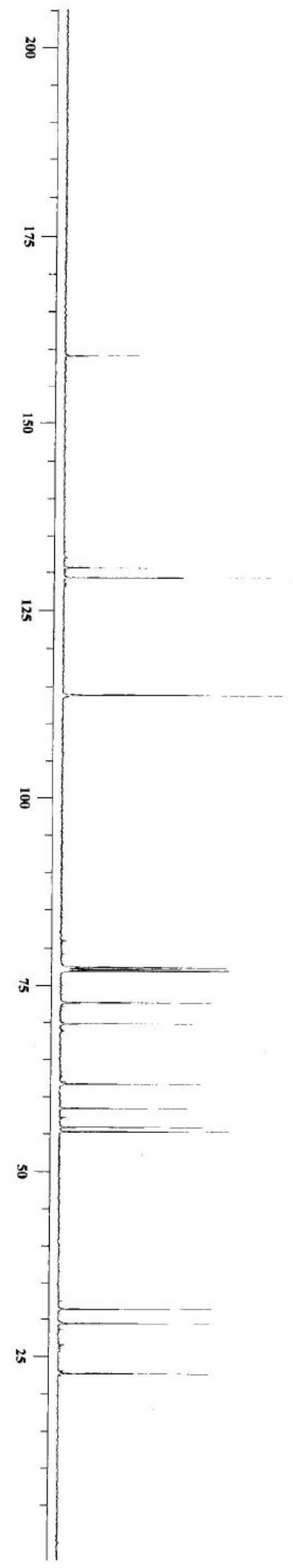

$-159.061$
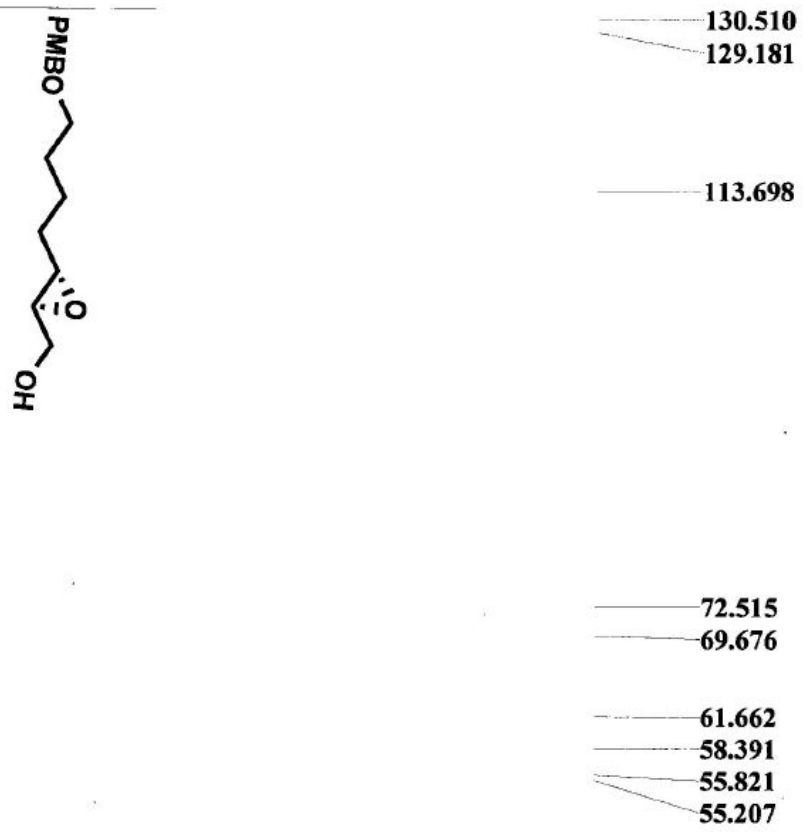

${ }^{13} \mathrm{C}$ NMR spectrum of $16\left(100 \mathrm{MHz}, \mathrm{CDCl}_{3}\right)$ 


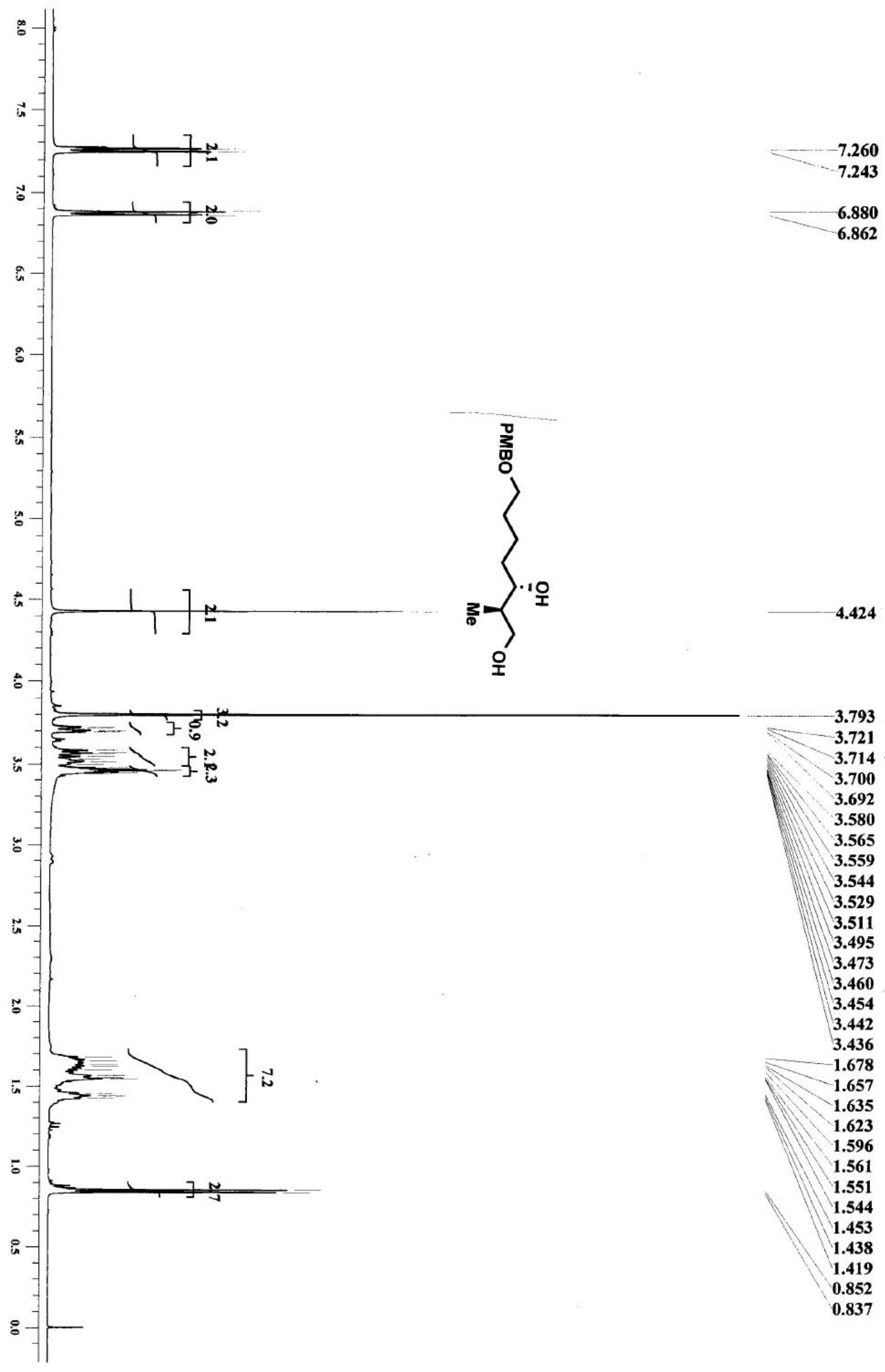

${ }^{1} \mathrm{H}$ NMR spectrum of $17\left(500 \mathrm{MHz}, \mathrm{CDCl}_{3}\right)$ 


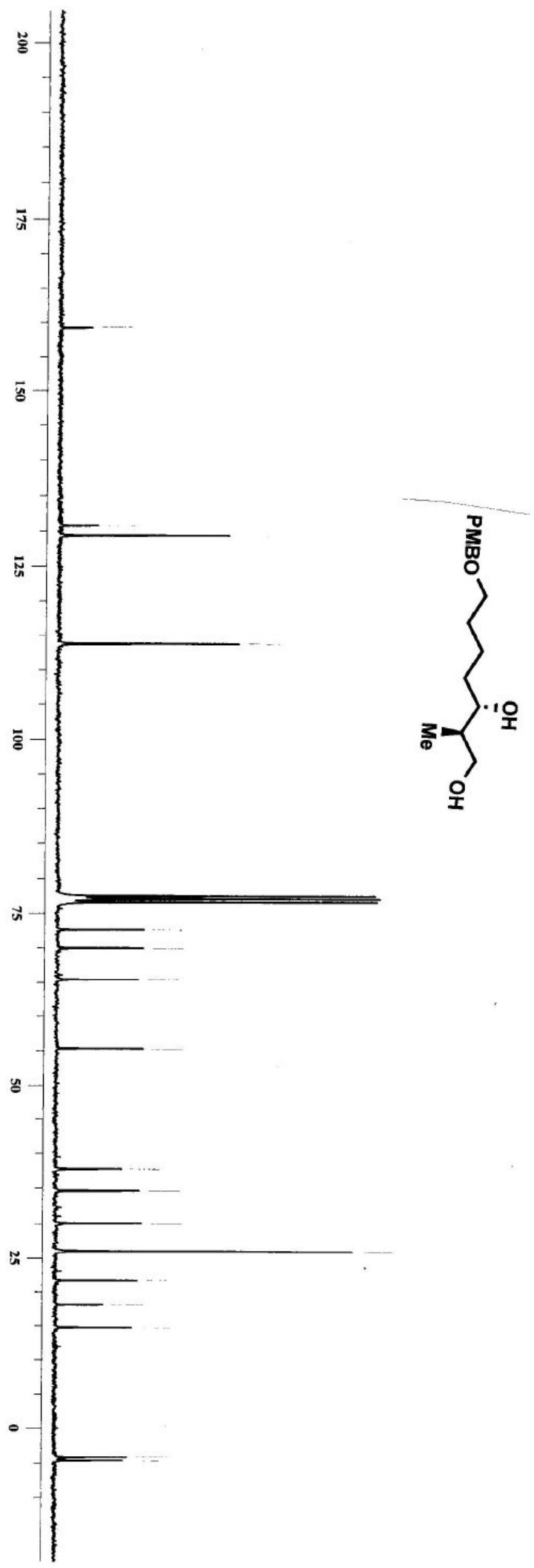

$-159.087$

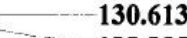

129.205

$-113.723$
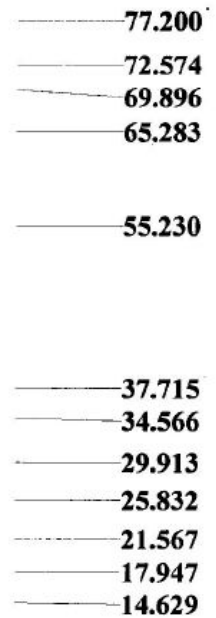

${ }^{13} \mathrm{C}$ NMR spectrum of $17\left(125 \mathrm{MHz}, \mathrm{CDCl}_{3}\right)$ 


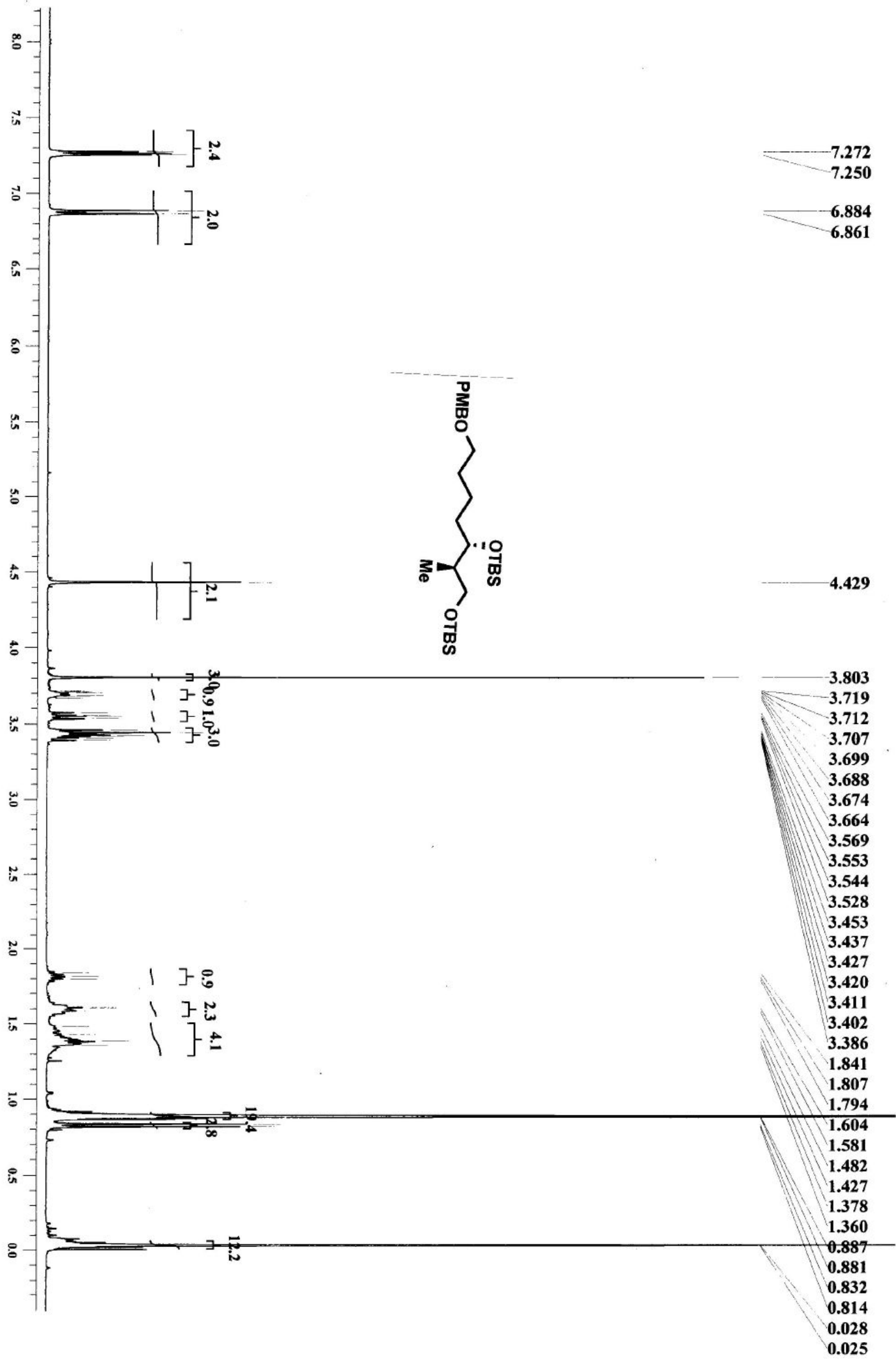

${ }^{1} \mathrm{H}$ NMR spectrum of $\mathbf{1 8}\left(500 \mathrm{MHz}, \mathrm{CDCl}_{3}\right)$ 


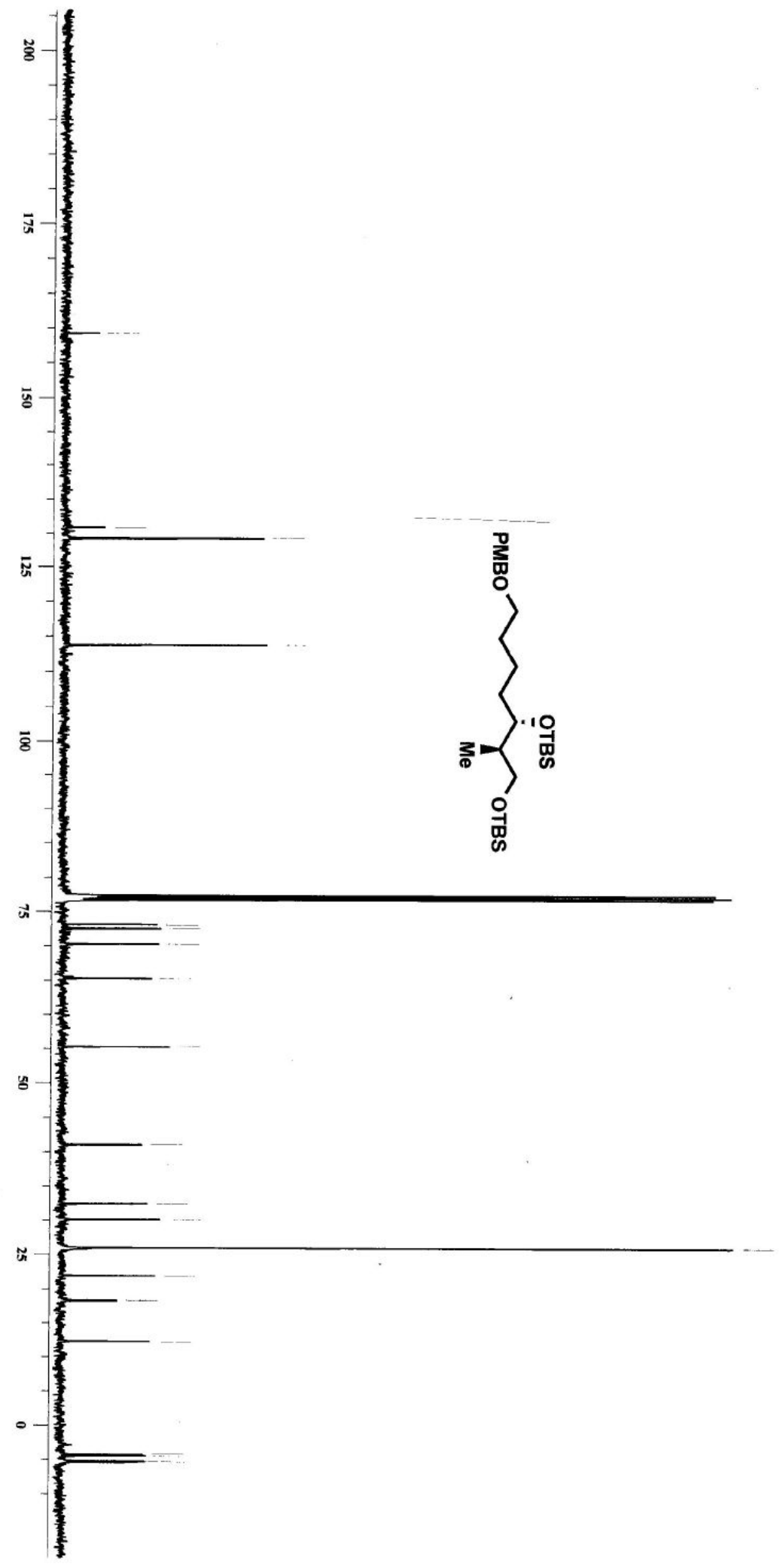

$-159.044$
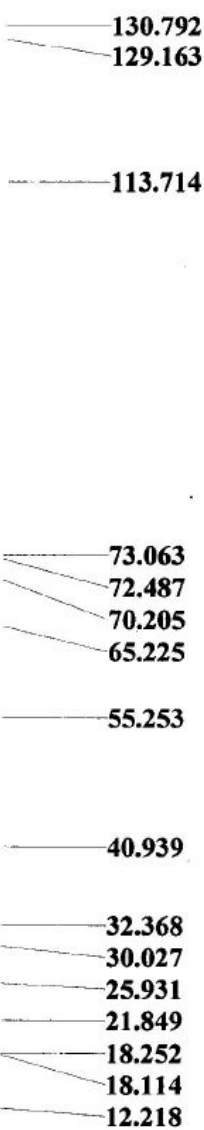

${ }^{13} \mathrm{C}$ NMR spectrum of $18\left(125 \mathrm{MHz}, \mathrm{CDCl}_{3}\right)$

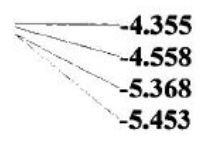




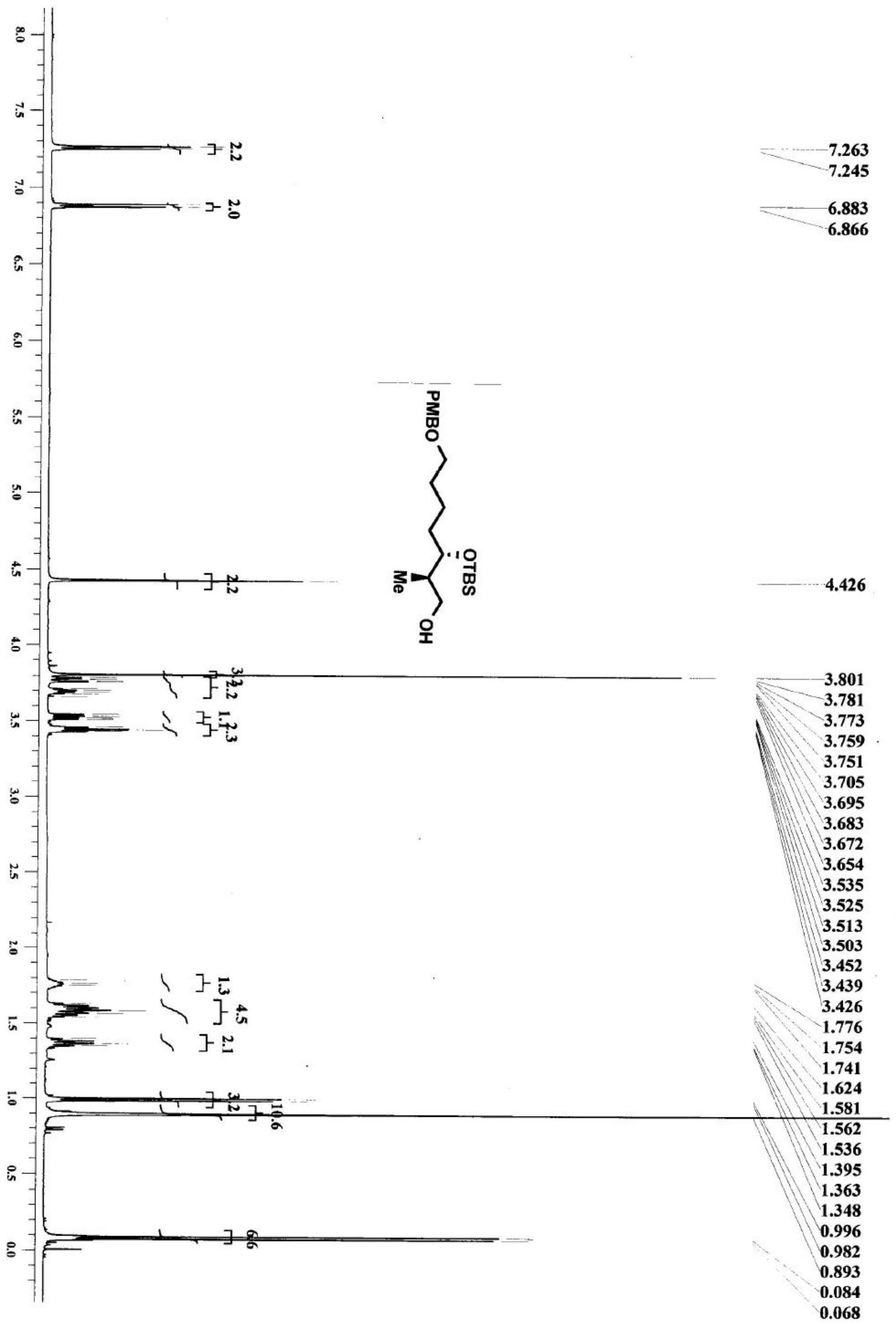

${ }^{1} \mathrm{H}$ NMR spectrum of $19\left(500 \mathrm{MHz}, \mathrm{CDCl}_{3}\right)$ 


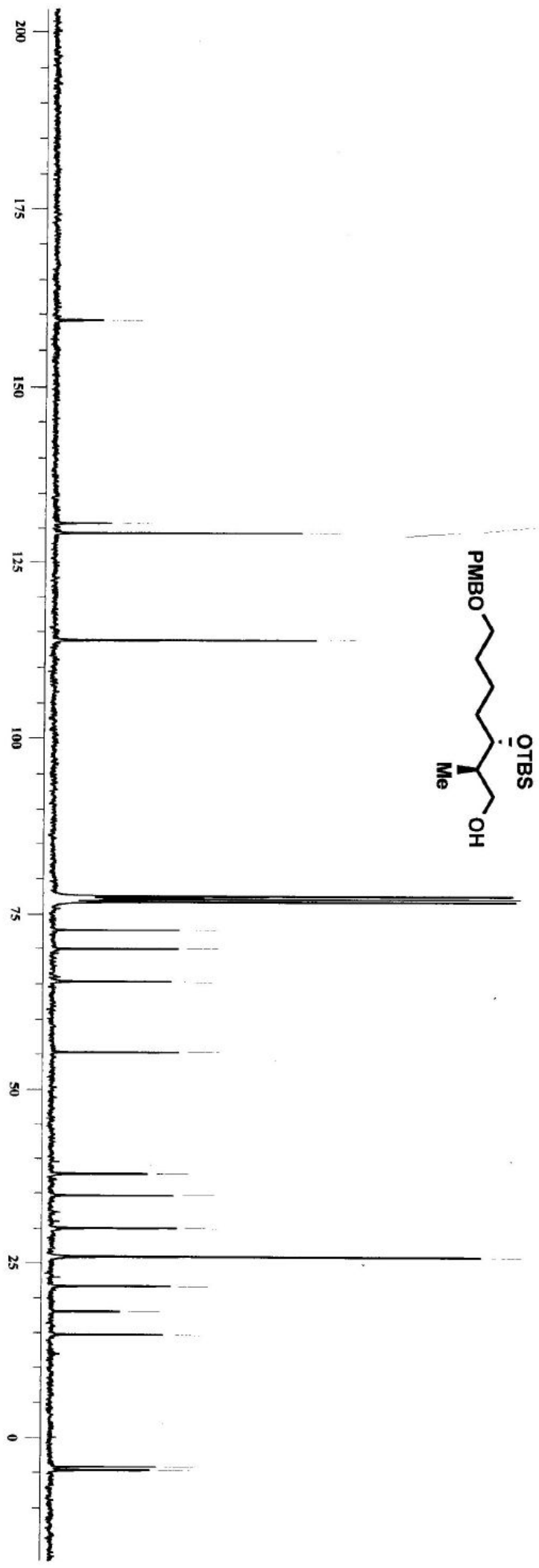

$-113.723$

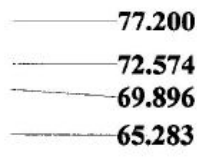

$\mathbf{5 5 . 2 3 0}$
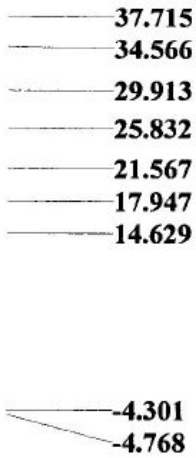

${ }^{13} \mathrm{C}$ NMR spectrum of $19\left(75 \mathrm{MHz}, \mathrm{CDCl}_{3}\right)$ 


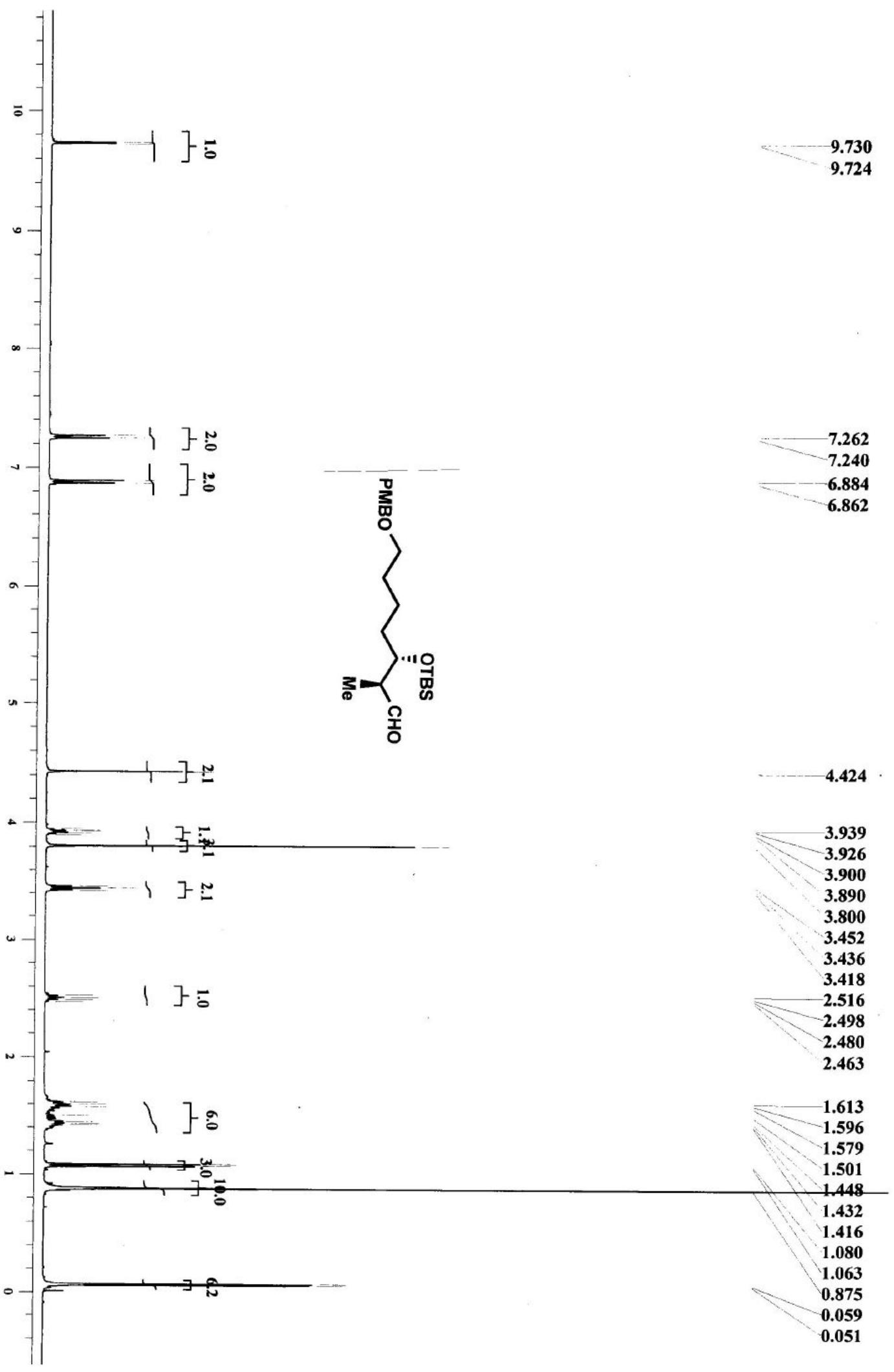

${ }^{1} \mathrm{H}$ NMR spectrum of $6\left(500 \mathrm{MHz}, \mathrm{CDCl}_{3}\right)$ 


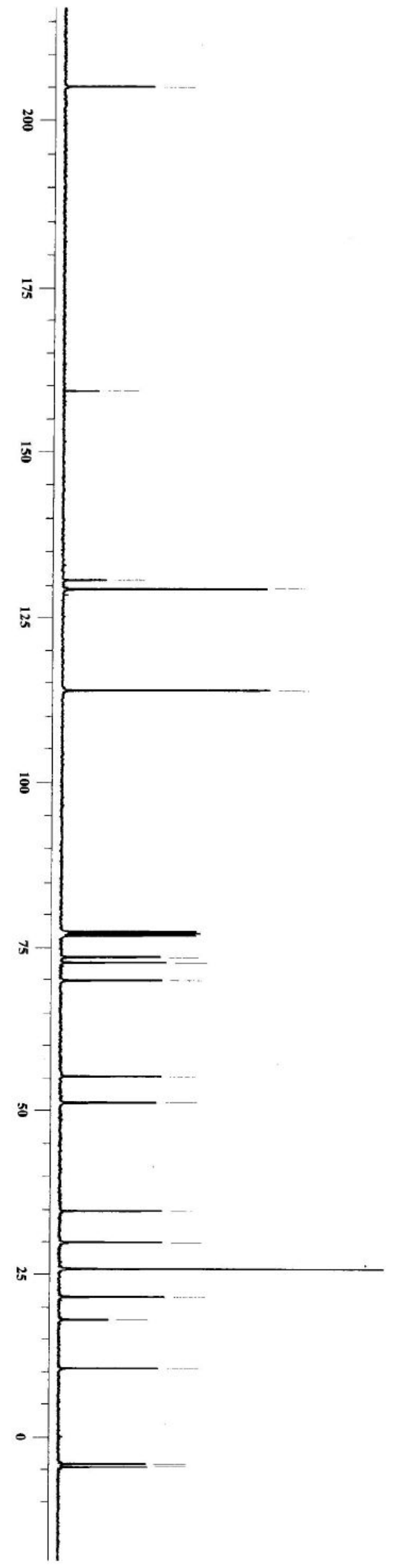

204.974

159.088
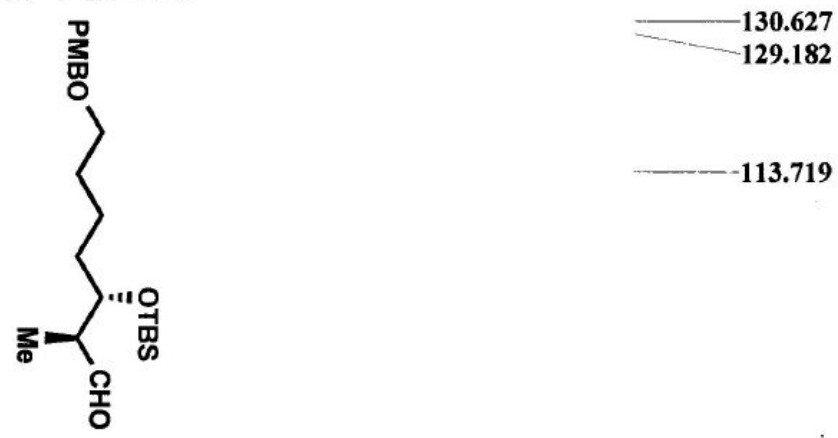

73.352

72.553

69.803

55.222

$\mathbf{5 1 . 0 3 0}$

$-34.584$

29.801

$-25.755$

21.483

$-18.004$

$-10.409$

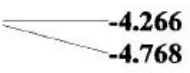

${ }^{13} \mathrm{C}$ NMR spectrum of $6\left(125 \mathrm{MHz}, \mathrm{CDCl}_{3}\right)$ 

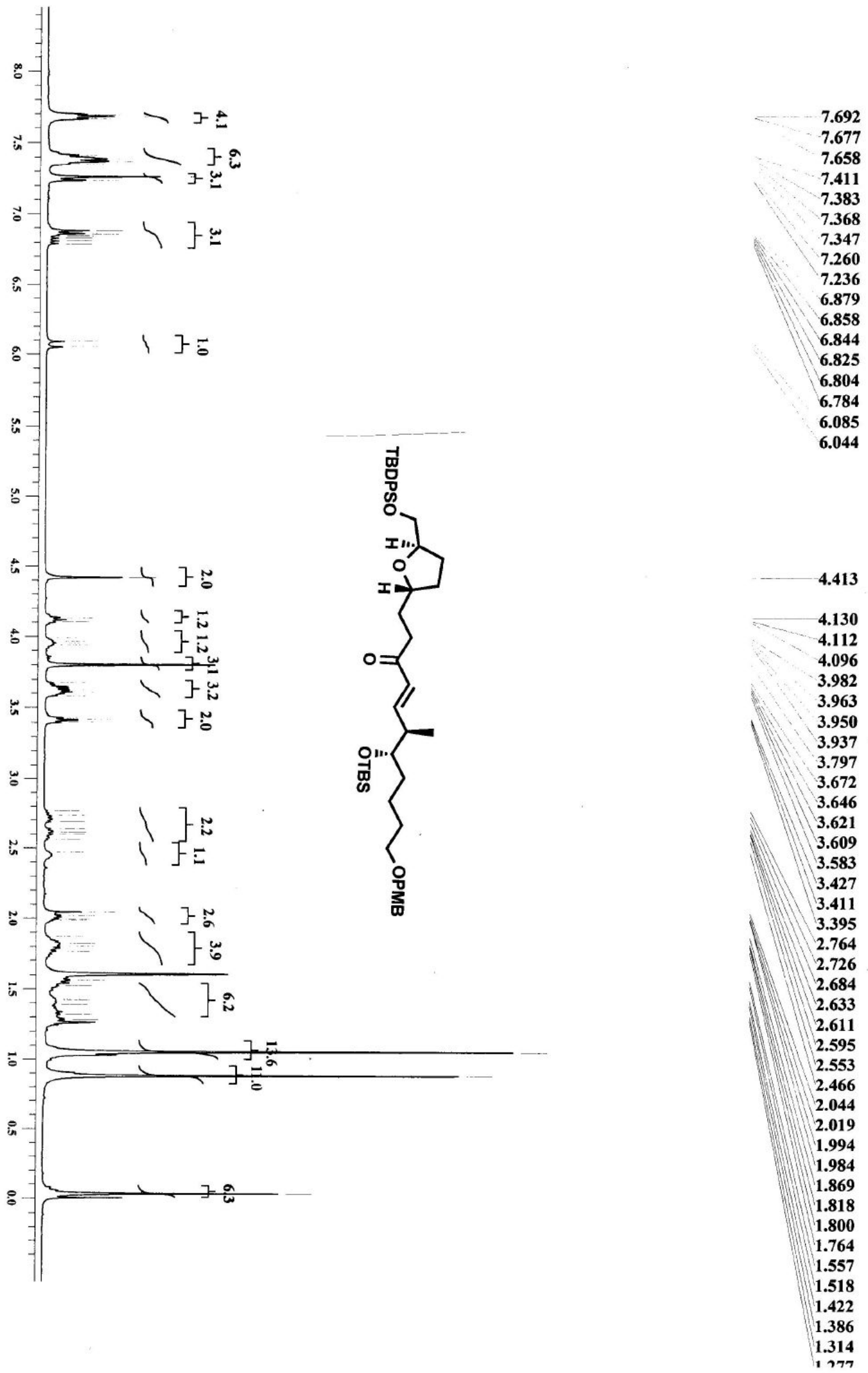

${ }^{1} \mathrm{H}$ NMR spectrum of $4\left(400 \mathrm{MHz}, \mathrm{CDCl}_{3}\right)$ 


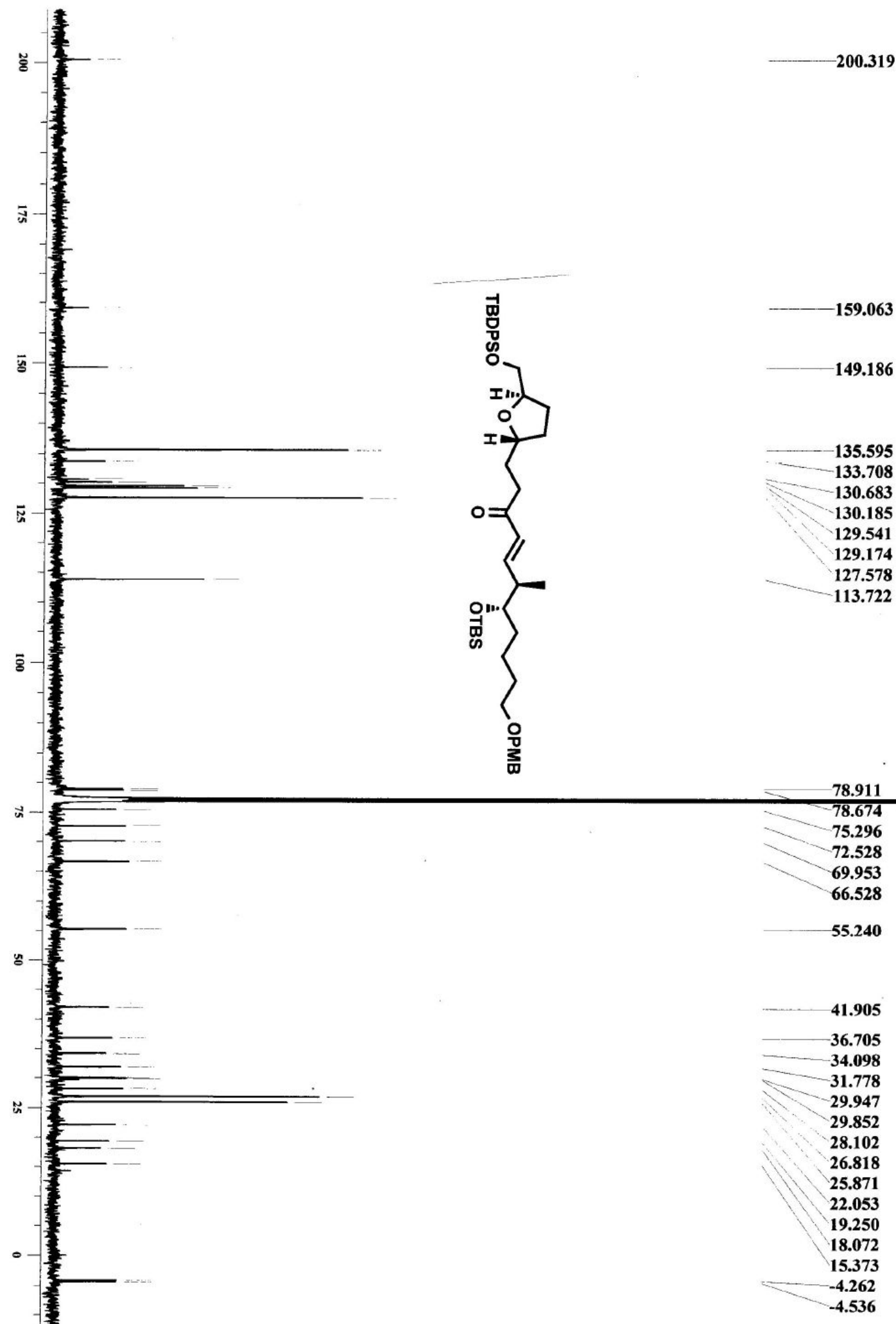

${ }^{13} \mathrm{C}$ NMR spectrum of $4\left(100 \mathrm{MHz}, \mathrm{CDCl}_{3}\right)$ 


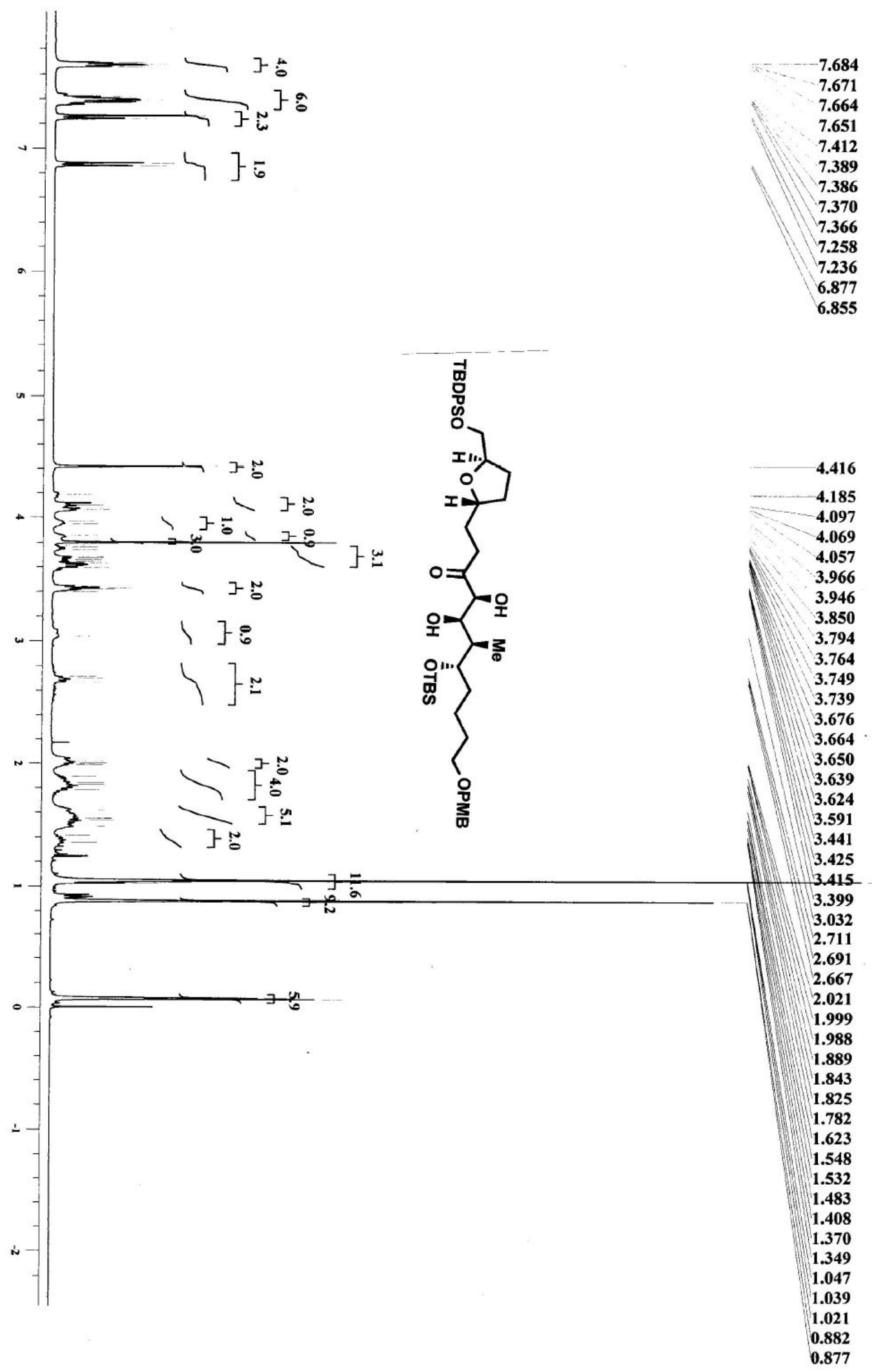

${ }^{1} \mathrm{H}$ NMR spectrum of $20\left(400 \mathrm{MHz}, \mathrm{CDCl}_{3}\right)$ 


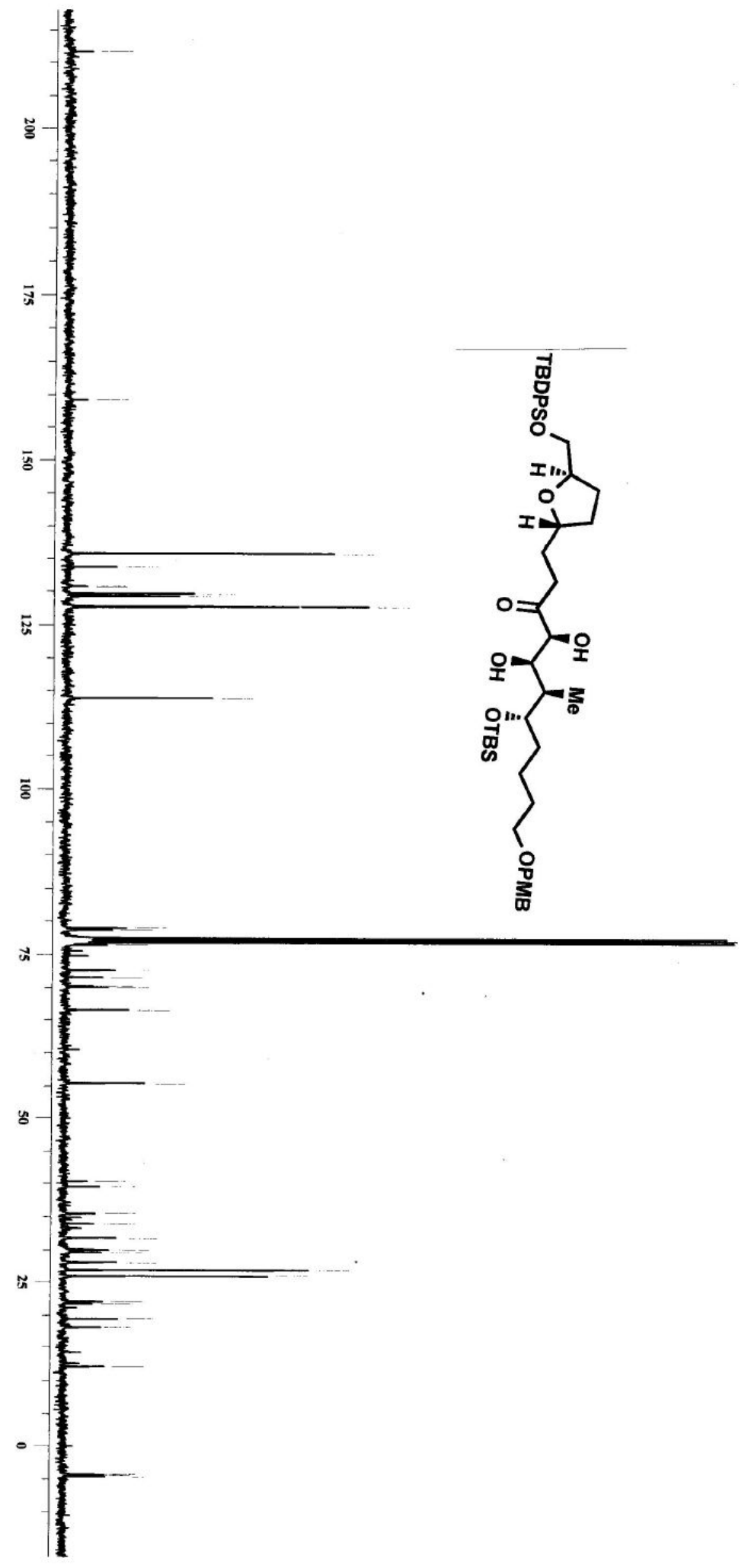

211.570

$-159.042$
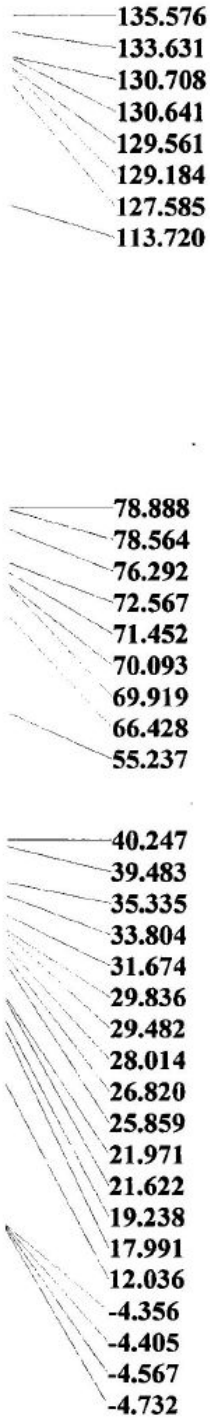

${ }^{13} \mathrm{C}$ NMR spectrum of $20\left(125 \mathrm{MHz}, \mathrm{CDCl}_{3}\right)$ 


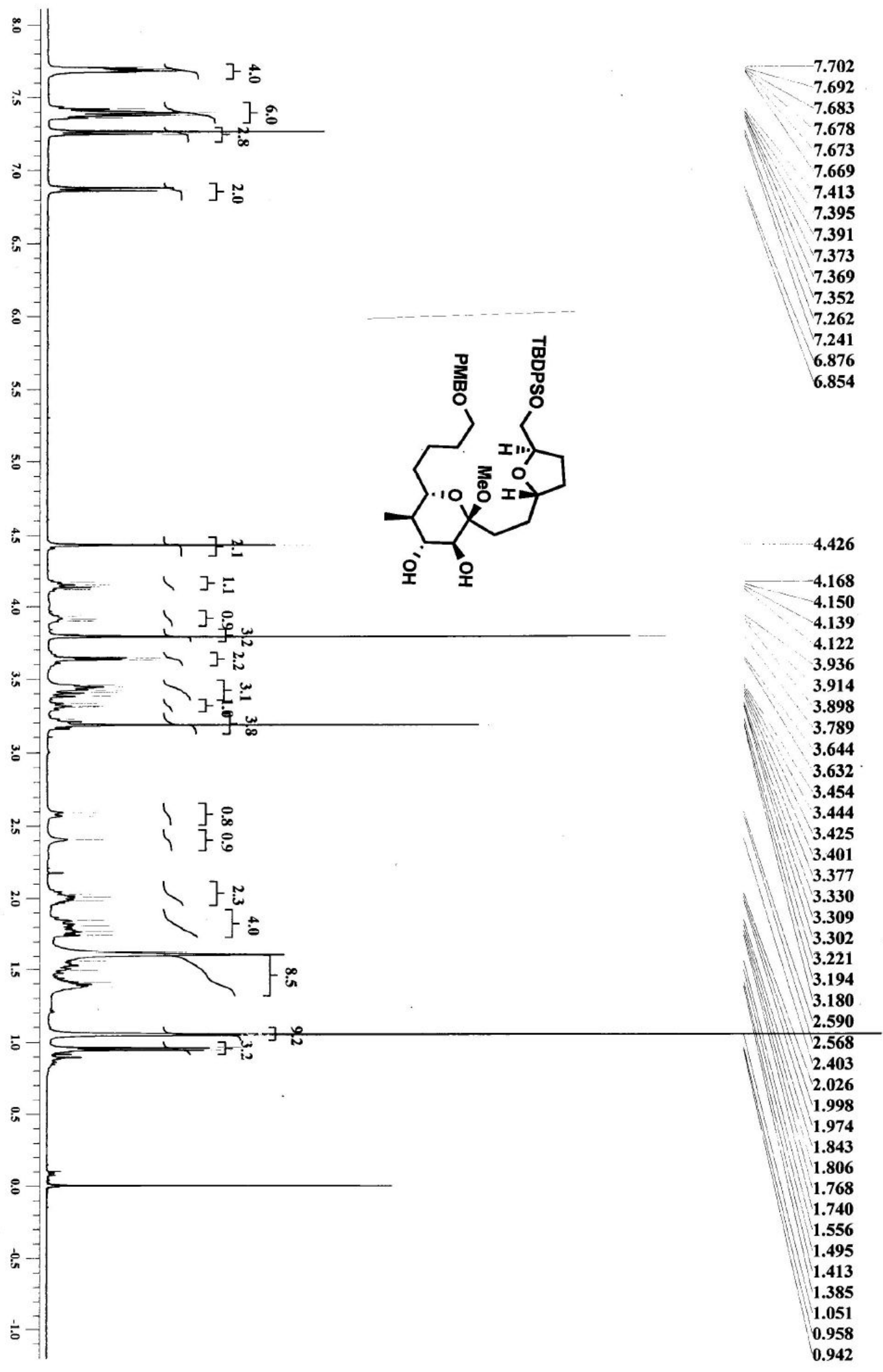

${ }^{1} \mathrm{H}$ NMR spectrum of $\mathbf{3}\left(500 \mathrm{MHz}, \mathrm{CDCl}_{3}\right)$ 


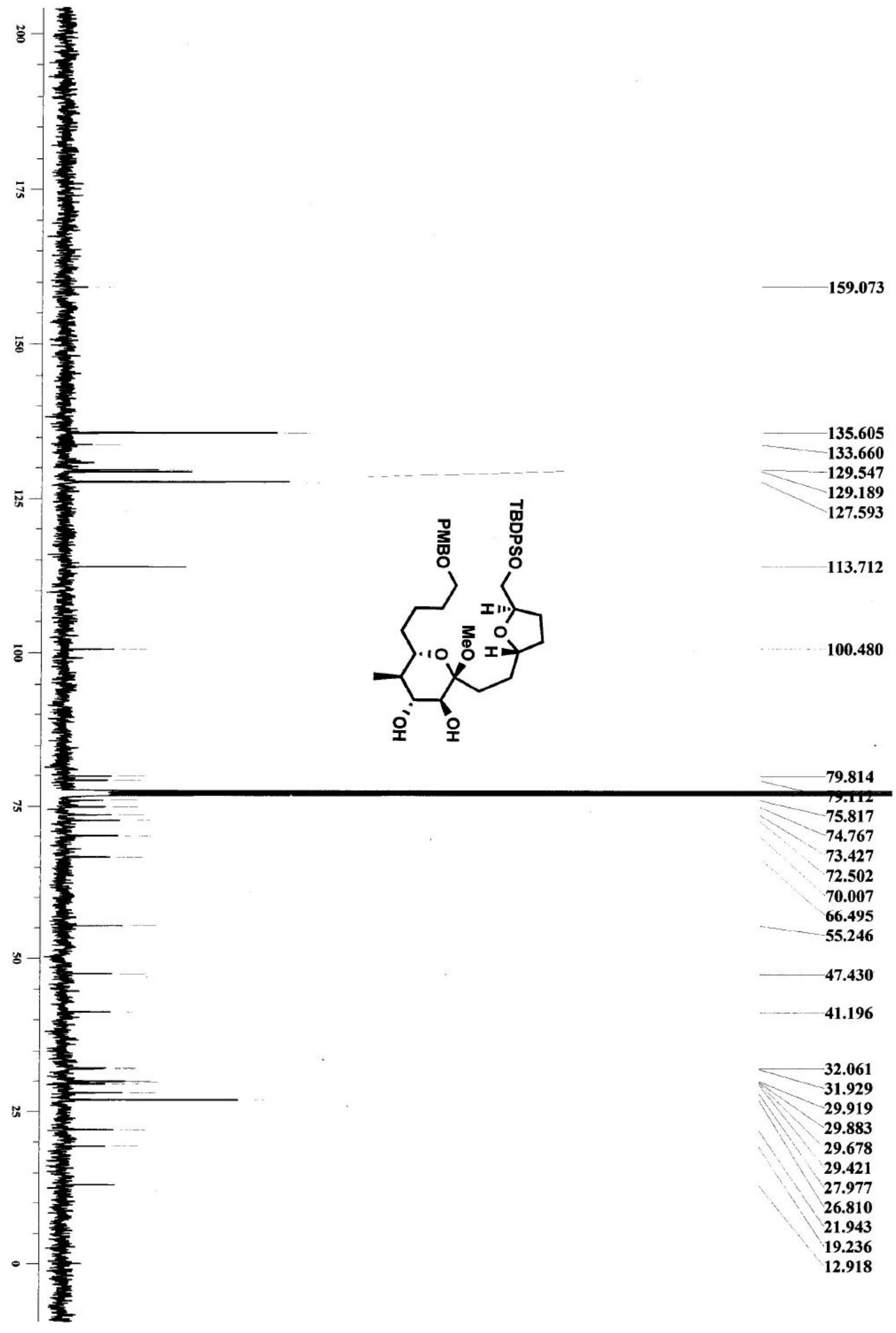

${ }^{13} \mathrm{C} \mathrm{NMR}$ spectrum of $5\left(125 \mathrm{MHz}, \mathrm{CDCl}_{3}\right)$ 


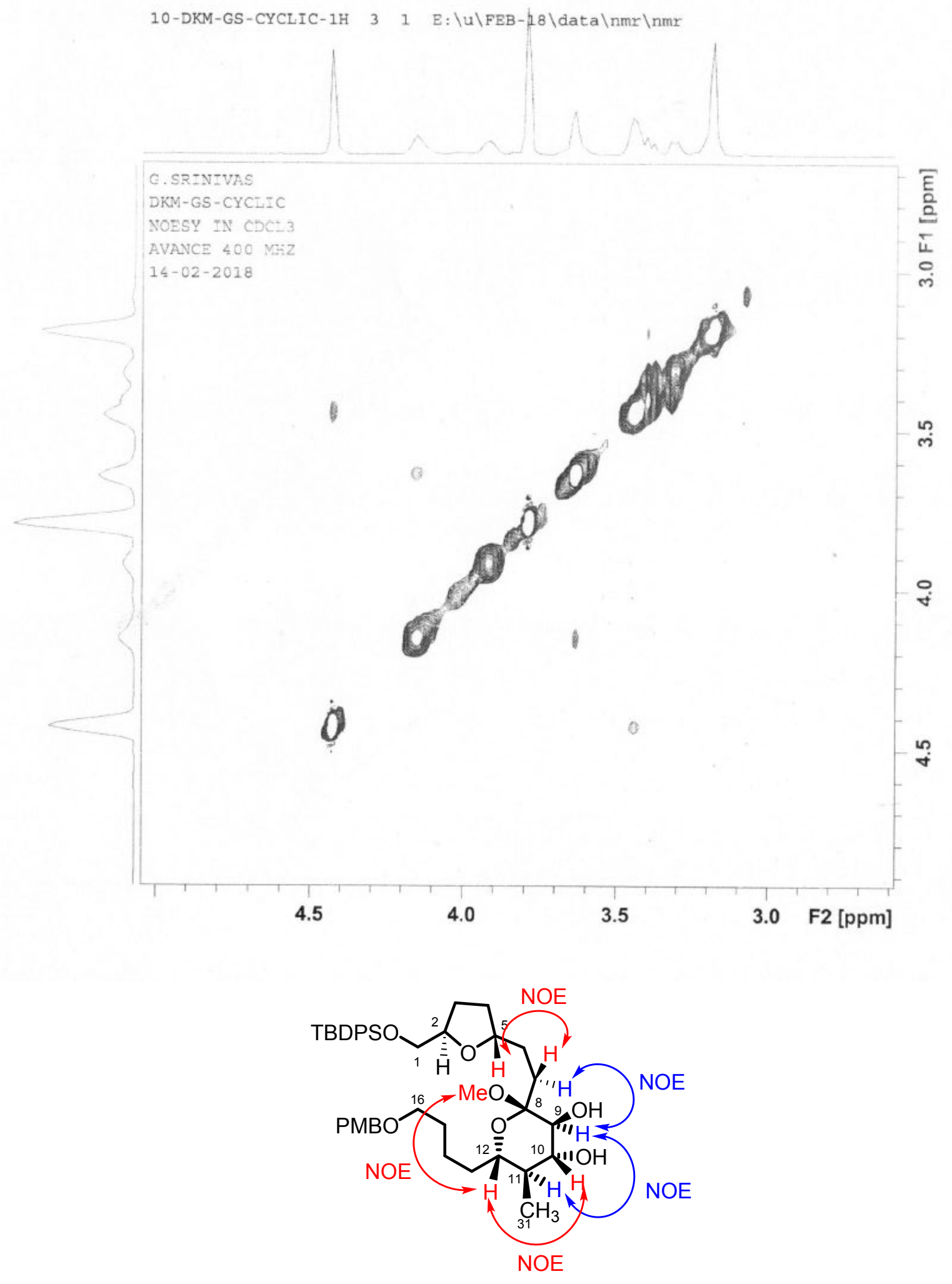

NOESY Spectrum of compound 3 (400 MHz, $\mathrm{CDCl}_{3}$ ) (2.5 to $5.0 \mathrm{ppm}$ ) 


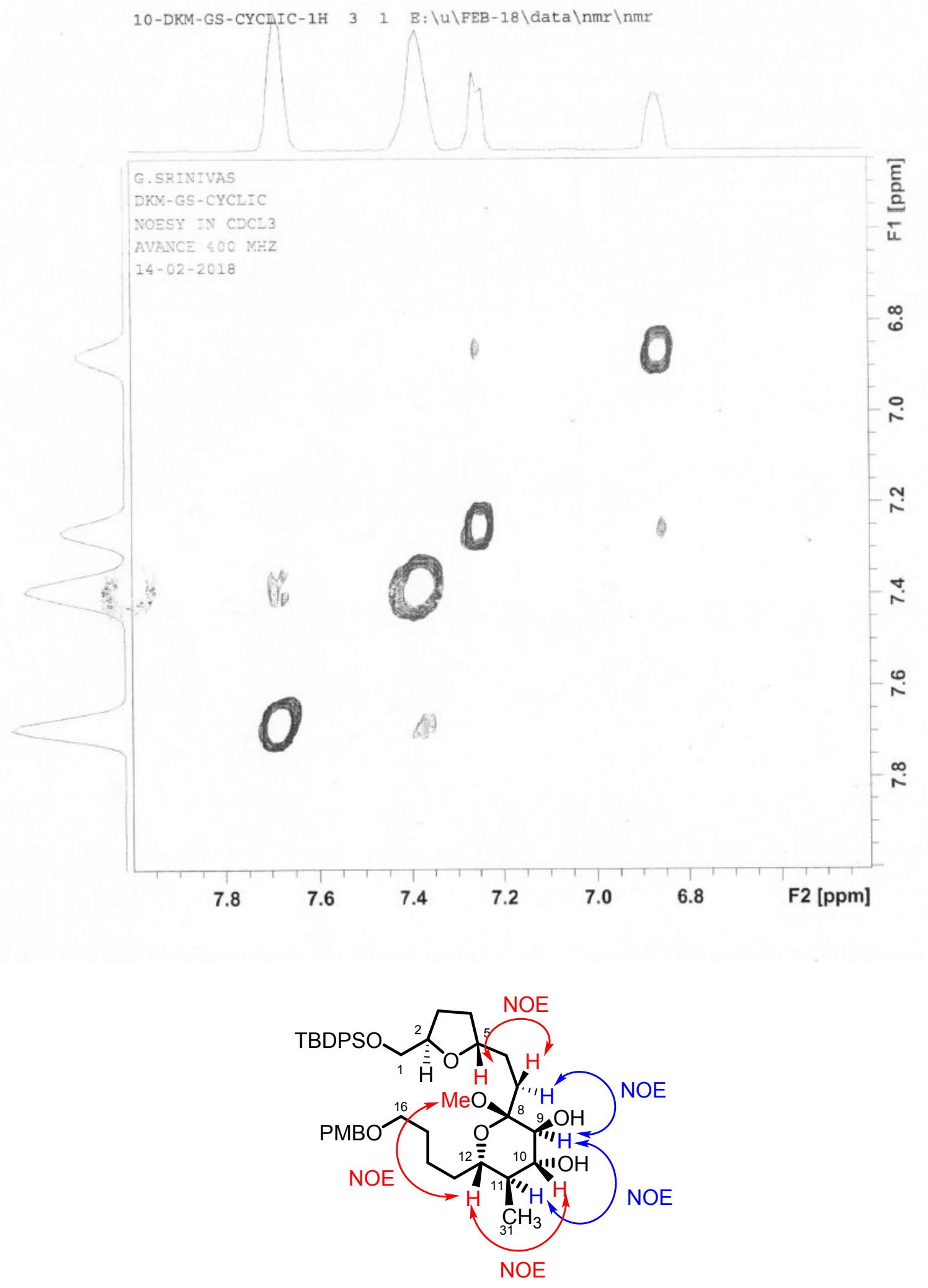

NOESY Spectrum of compound 3 (400 MHz, $\mathrm{CDCl}_{3}$ ) (6.0 to $\left.8.0 \mathrm{ppm}\right)$ 

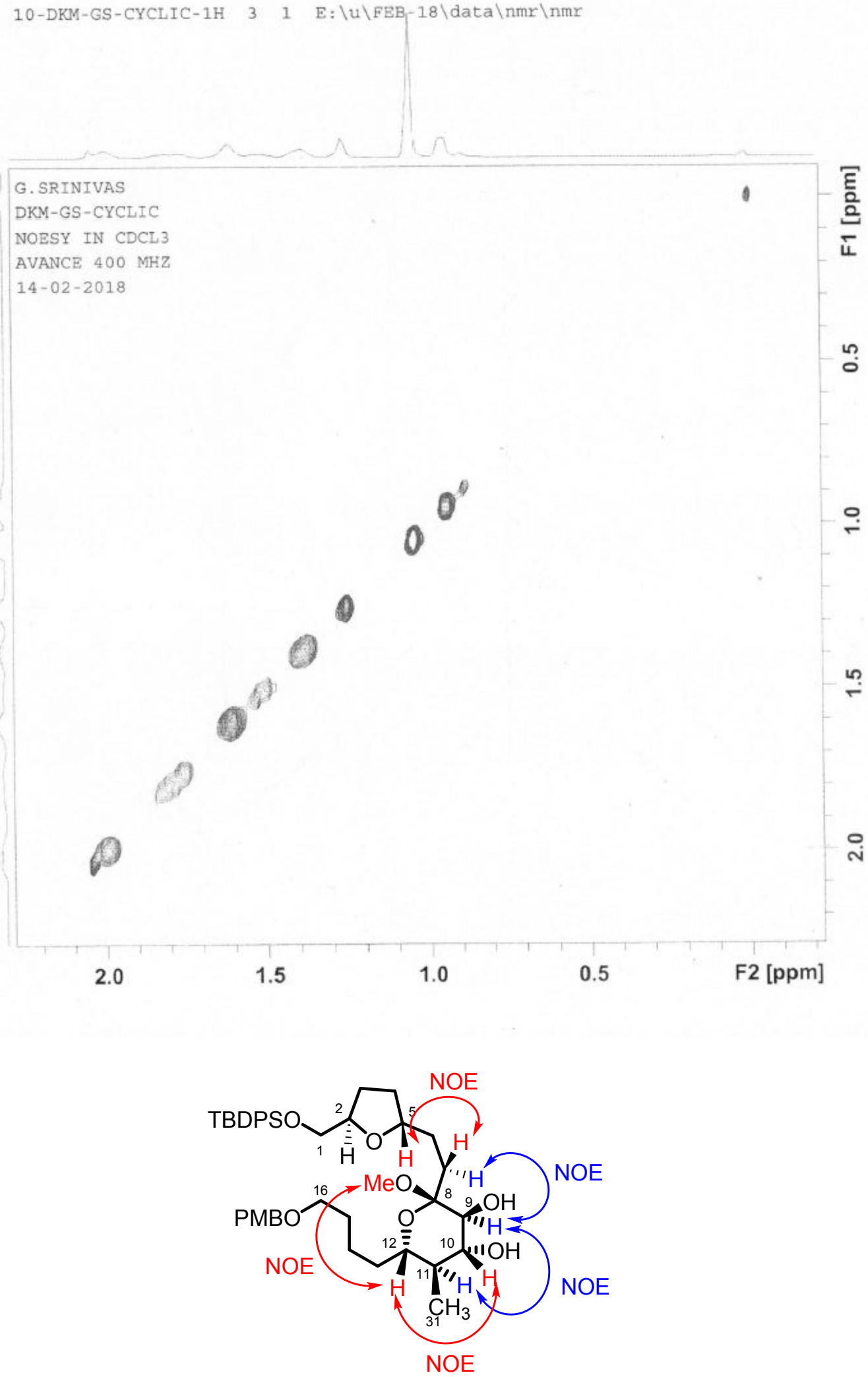

NOESY Spectrum of compound $3\left(400 \mathrm{MHz}, \mathrm{CDCl}_{3}\right)$ (-0.2 to $\left.2.2 \mathrm{ppm}\right)$ 

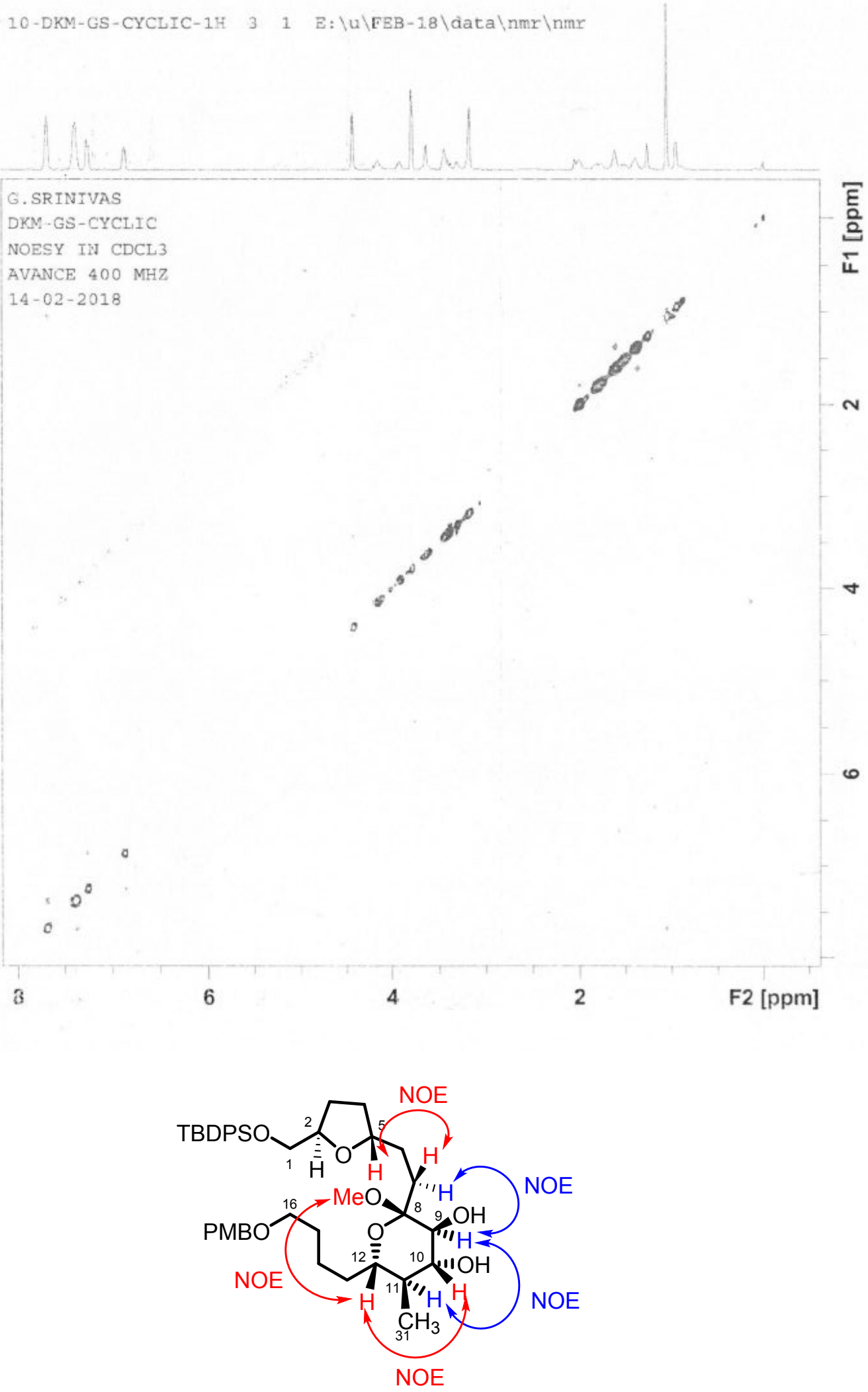

NOESY Spectrum of compound 3 (400 MHz, $\mathrm{CDCl}_{3}$ ) (Full Spectrum) 